\title{
Functional Extension of High-Speed AFM for Wider Biological Applications
}

Takayuki Uchihashi ${ }^{1,2,3}$, Hiroki Watanabe ${ }^{1}$, Shingo Fukuda ${ }^{1}$, Mikihiro Shibata ${ }^{1}$ and Toshio Ando ${ }^{1,2,3}$

${ }^{1}$ Department of Physics, Kanazawa University, Kanazawa 920-1192, Japan.

${ }^{2}$ Bio-AFM Frontier Research Center, Kanazawa University, Kanazawa 920-1192, Japan.

${ }^{3}$ CREST/JST, Tokyo 102-0075, Japan.

High-speed atomic force microscopy (HS-AFM) has been established and used, which can visualize biomolecules in dynamic action at high spatiotemporal resolution without disturbing their function. Various studies conducted in the past few years have demonstrated that the dynamic structure and action of biomolecules revealed with HS-AFM can provide greater insights than ever before into how the molecules function. However, this microscopy has still limitations in some regards. Recently, efforts have been carried out to overcome some of the limitations. As a result, it has now become possible to visualize dynamic processes occurring even on live cells and perform simultaneous observations of topographic and fluorescent images at a high rate. In this review, we focus on technical developments for expanding the range of objects and phenomena observable by HS-AFM as well as for granting multiple functionalities to HS-AFM.

(C) 2015. This manuscript version is made available under the Elsevier user license http://www.elsevier.com/open-access/userlicense/1.0/ 


\section{Introduction}

Since the invention of atomic force microscopy (AFM) [1], increasing its imaging rate has been one of the major technical challenges for enhancing the usefulness of AFM. The materialization of high-speed AFM (HS-AFM) required various developments and breakthroughs, as described below, while it was expected to bring a great impact on various fields of science and technology. Biological science in particular would most efficaciously receive a great deal of benefit from the materialization. This is because the direct observation of biological molecules in dynamic action at high spatiotemporal resolution certainly facilitates our detailed understanding of their functional mechanism. Driven by this motivation, Paul Hansma's group and Ando's group independently embarked on the development of HS-AFM more than two decades ago. In the early stage, efforts were focused on the developments of a fast scanner [2-4], small cantilevers with a high resonant frequency in water and a small spring constant [3-7], an optical beam deflection (OBD) detector that detects the deflection of a small cantilever [3,5], and a fast amplitude detector that quickly converts the deflection signal of the oscillating cantilever to its amplitude signal $[3,8]$. By assembling these (or some of these) devices, prototypic HS-AFM instruments employing the tapping mode were built around 2000 and shown to be able to capture images of protein molecules at a much higher rate than before [3,9]. However, it was evident that the feedback bandwidth was still insufficient and therefore the imaging rate was not high enough or the protein molecules were damaged when imaged too fast. Then, Ando's group has further endeavored to enhance the capacity of HS-AFM by developing various techniques such as an active damping technique that suppresses the Z-scanner's unwanted vibrations as well as enhances its response speed [10], a new scheme for the proportional-integral-derivative (PID) control (referred to dynamic PID control) that can make high-speed imaging compatible with low-invasiveness to fragile molecules and weak intermolecular interactions [11], a technique to compensate for drift of the cantilever excitation power [11] and a fast phase detector [12]. Moreover, Ando's group has developed smaller cantilevers in collaboration with Olympus, a more robust fast scanner and a lower-noise, fast amplitude detector [13]. Finally, around 2008, HS-AFM of practical use was established that achieved a feedback bandwidth of $\sim 100$ $\mathrm{kHz}$ and therefore could capture images of protein molecules at sub-100 ms temporal resolution, without disturbing their function [13].

Since the establishment of this HS-AFM, its excellent performance has been demonstrated by imaging studies on various proteins in dynamic action. For example, conformational dynamics of a photo-activated proton pump, bacteriorhodopsin [14-16] 
and a rotary-motor protein, $\mathrm{F}_{1}$-ATPase [17], dynamic processes of linear-motor proteins, myosin V [18], cellulose [19-21] and chitinase [22], dynamic protein-protein interactions $[23,24]$, dynamics occurring in the crystal lattices of a soluble protein and membrane protein $[25,26]$, and wiggling motion of intrinsically disordered proteins $[27,28]$ have been successfully visualized (see Reviews [29,30]). The exquisite dynamic images not only provided corroborative "visual evidence" for previous inferences but also solved long-standing questions that had previously been difficult or impossible to address by other approaches. Thus, HS-AFM has now become holding an indisputable position as a new imaging tool in biological science.

Nevertheless, the current HS-AFM that adopts the sample stage-scanning mode has limitations in some regards. To achieve the high scan rate and minimize the hydrodynamic effect of fast sample-stage movement on the cantilever behavior, a small sample stage (a glass rod with dimensions of 1-1.5 $\mathrm{mm}$ in diameter and $2 \mathrm{~mm}$ in height) attached to the Z-scanner has to be used [13]. This small size restricts the range of specimens to be placed on the sample stage. To remove this restriction, HS-AFM should adopt the tip-scanning mode, in which the cantilever chip is scanned in the three dimensions. Tip-scanning HS-AFM has another advantage that optical microscopy techniques can easily be incorporated to the system, including fluorescence microscopy techniques such as a total internal reflection fluorescence microscopy (TIRFM) technique. AFM that visualizes the surface structure of objects lacks the capability of identifying the target molecules when the molecules have no distinct structural features or exist in a crowded system containing multiple species, whereas fluorescence microscopy that detects fluorescence emitted from fluorescent dye-labeled molecules can identify the labeled molecules but can neither reveal their structures nor detect the surrounding non-labeled molecules and environment. As such, the combination of the two microscopy techniques would redeem their weaknesses. Another restriction of the current HS-AFM system is that the maximum scan range is limited approximately to 2 $\times 4 \mu \mathrm{m}^{2}$, which does not allow us to observe dynamic events occurring in large objects such as live bacterial and mammalian cells. To remove this restriction, we need to develop a wide-area scanner as well as an active damping technique to suppress unwanted vibrations that are easily generated by fast scanning of such a large scanner having low resonant frequencies.

To make HS-AFM more versatile for the use in more biological applications, these new technical developments are essential. In this review, we describe recent technical advances that have successfully removed the restrictions mentioned above $[31,32]$. Applications studies of this state-of-art HS-AFM have not yet been explored as these 
advances were made very recently. However, we also describe biological application studies and show their results that have demonstrated the new capability, functionality and potential of the state-of-art HS-AFM.

\section{Tip-scanning HS-AFM Combined with TIRFM}

Fluorescence microscopy is a proven technique to visualize biological specimens over a wide range from single molecules to cells and tissues [33-38]. The most recent advance in super resolution fluorescence microscopy has made it possible to resolve, beyond the diffraction limit of light, the positions of individual fluorophores that are closely located (less than $\sim 20 \mathrm{~nm}$ apart) in biological structures [39]. Fluorescence microscopy can detect small molecules like fluorescently labeled nucleotides, whereas AFM cannot do so. Furthermore, it is even possible in fluorescence microscopy to identify two or three species of molecules in a sample containing multiple components by specific labeling of the molecules with differently colored fluorescent dyes or by the construction of fluorescent proteins with different colors [40,41]. However, in fluorescence microscopy the structure of labeled molecules themselves cannot be revealed even with super resolution since it only detects the fluorescent light emitted from the labeled molecules. Therefore, AFM and fluorescence microscopy are mutually complementary and hence their combined system would be a valuable, versatile tool in biological studies. Thus far, various developments of AFM systems combined with fluorescence microscopy have been reported [42-45]. Such a combined system is also developed for HS-AFM [46,47]. However, in previous combined systems, topographic AFM and single-molecule fluorescence images cannot be captured simultaneously at high spatiotemporal resolution.

\section{2-1. Laser Tracking Method}

The major technical challenge in developing a HS-AFM system combined with an optical microscope is that the tip-scanning mode has to be adopted, instead of the sample-scanning mode that has always been used for HS-AFM [3,5]. In the first place, this is because the sample stage must be stationary. Otherwise, the optical image oscillates during HS-AFM imaging. Secondly, this is because the sample-stage scanner is incompatible with the objective lens for optical microscopy when the combined system is designed so that the cantilever does not interfere with optical imaging. In tip-scanning AFM, the OBD detector has to scan its laser beam to have the focused spot always on a given positon within the cantilever under scan. As a matter of course, the OBD detector itself can be scanned in synchrony with XY scanning of the cantilever but 
such a heavy system cannot be scanned fast. Mechanisms for this laser beam tracking have been designed and installed in conventional slow AFM systems, where the laser diode and focusing lens are integrated into a piezo-tube scanner to which the cantilever chip is fixed [48], or mirrors and a focusing lens are integrated to the cantilever chip-scanner [49]. However, these designs cannot be used for HS-AFM. The piezo-tube scanner is impossible to be used for HS-AFM; when its size is minimized to achieve high resonant frequencies, significant cross-talk between the three scan axes occurs. Also, multiple mirrors cannot be equipped in a small-sized fast scanner. A tip-scanning HS-AFM system is also reported that uses a focusing lens embedded in the fast $\mathrm{XY}$-scanner to scan the laser beam in the $\mathrm{X}$ - and Y-directions [46]. The small single lens used therein has a high numerical aperture (NA) and a short working distance, and therefore, can focus the laser beam to a small spot (2-4 $\mu \mathrm{m}$ in diameter), comparable to the width of small cantilevers $(\sim 2 \mu \mathrm{m})$. However, when the Z-scanner is scanned more than $\sim 150 \mathrm{~nm}$, the laser beam is certainly defocused on the cantilever, resulting in a larger spot size and hence a significant error in the amplitude detection .

Considering these issues, we adopted a two-dimensional mirror tilter to scan the laser beam from the OBD detector in the X-and Y-directions [31]. The mirror is a dichroic type with $>90 \%$ transmission at $400-690 \mathrm{~nm}$ and $>95 \%$ reflection at $700-1150$ $\mathrm{nm}$. It reflects the incident laser beam of wavelength $>700 \mathrm{~nm}$ and guides the reflected laser beam onto an objective lens with a long working distance-of - $24 \mathrm{~mm}$ (CFI L Plan EPI ELWD, $\times 20, \mathrm{NA}, 0.35$, Nikon), making a small focused spot $(2-3 \mu \mathrm{m}$ in diameter) on the small cantilever. The two directional angles of the normal line to the mirror plane are changed in synchrony with XY-scanning of the cantilever, as depicted in Fig. 1(a). In addition to the dichroic mirror, the mirror tilter is composed of two base plates and four piezo actuators with the maximum displacement of $\sim 3 \mu \mathrm{m}$ at $100 \mathrm{~V}$, as shown in Figs. 1(b) and (c). The dichroic mirror is supported by the two X-piezoactuators glued on the base plate 2 through a beveled block. This whole unit is further supported by the two Y-piezoactuators glued on the base plate 1. The respective pairs of piezoactuators are displaced in the opposite directions, by which the respective tilt angles of the dichroic mirror are scanned. The maximum change of the tile angle was estimated to be approximately $\pm 4.8 \times 10^{-4}$ radian for $\mathrm{X}$-directions. This angle change corresponds to the maximum lateral displacements of the focused spot, approximately $19 \mu \mathrm{m}$, considering the focal length of the objective lens, $20 \mathrm{~mm}$. The actual maximum displacement of the focused spot measured using a CCD camera was about $20 \mu \mathrm{m}$, in good agreement with the estimation.

The laser beam reflected from the cantilever is collimated by the objective lens, 
reflected by the dichroic mirror, and then guided onto the two-segmented Si PIN photodiode sensor through the $\lambda / 4$ plate, polarization beam splitter and the focusing lens (see Fig. 3). Therefore, the incident position of the reflected laser on the photodiode sensor is altered by scanning of the mirror tilter, and therefore, the DC component of the differential signal that is output from the photodiode sensor is also altered. However, its AC component corresponding to the cantilever oscillation amplitude is unaltered. In tapping-mode HS-AFM only the AC component is used for imaging, and therefore, this alteration of the DC component does not affect HS-AFM imaging.

The mirror tilter is relatively heavy and therefore has low resonant frequencies that would easily be excited by its fast scanning. When the X-tilter was scanned at various frequencies, the frequency response of the tilting motion showed the first resonant peak around $2.2 \mathrm{kHz}$, which was followed by a few peaks at higher frequencies. These resonant vibrations distort and delay the mechanical response of the X-tilter as shown in Fig. 1(d). This problem was solved by an inversion-based feed-forward damping [50]; an isosceles triangle wave as an original driving signal was digitally filtered, as follows. The isosceles triangle wave is characterized by amplitude $X_{0}$ and fundamental angular frequency $\omega_{0}$. The Fourier transform of its series of waves is given by

$$
F(\omega)=2 \pi X_{0}\left[\frac{1}{2} \delta(\omega)-\frac{2}{\pi^{2}} \sum_{k=-\infty}^{+\infty} \frac{1}{k^{2}} \delta\left(\omega-k \omega_{0}\right)\right] \quad(k: o d d) .
$$

The compensated signal $D_{\mathrm{X}}(t)$ to drive the X-tilter that is characterized by the transfer function $G(i \omega)=g(\omega) \times e^{i \theta(\omega)}$ (corresponding to the frequency response; $g$, gain; $\theta$, phase) is given by the inverse Fourier transform of $F(\omega) / G(i \omega)$, which is expressed as

$$
D_{X}(t)=\frac{1}{\alpha} \times\left[\frac{X_{0}}{2}-\frac{4 X_{0}}{\pi^{2}} \sum_{k=1}^{+\infty} \frac{1}{k^{2}} \frac{1}{G\left(i k \omega_{0}\right)} \cos \left(k \omega_{0} t\right)\right] \quad(k: o d d),
$$

where $\alpha(\mathrm{nm} / \mathrm{V})$ is the extension coefficient of the piezoactuators. Practically, the sum of the first $\sim 10$ terms in the series of Eq. (2) is sufficient. When driven by this compensated signal of $1 \mathrm{kHz}$ shown with the black line in Fig. 1(e), the X-tilter moves smoothly in an isosceles triangle wave, as shown by the red line in Fig. 1(e). This driving frequency corresponds to an imaging rate of 10 frames/s (fps) for 100 scan lines in the Y-direction. The mechanical responses of the Y-tilter also showed the first resonant peak around $2.2 \mathrm{kHz}$ and several larger peaks at higher frequencies. The Y-tilter is driven by a saw-tooth wave containing a precipitous downward regime for the tip being returned to the scan origin, which generates significant vibrations. To avoid fatal vibration generation, the Y-tilter scan was slowed down only in the downward regime. The Y-tilter moved smoothly by this driving. This altered driving only slightly 
$(\sim 5 \%)$ lowers the imaging rate.

The performance of the laser-beam tracking system was assessed by monitoring the cantilever oscillation amplitude when the oscillating cantilever was scanned laterally over a $650 \times 650 \mathrm{~nm}^{2}$ area without feedback scan in the Z-direction, while the tip was not in contact with the substrate surface. Figure 2(a) shows an amplitude image acquired at $1 \mathrm{fps}$ when the laser beam tracking system was switched off. The significant image variation seen in Fig. 2(a) indicates that the optical lever sensitivity varies due to positional shift of the cantilever relative to the stationary laser spot focused on it. The cross-section profile along the red line in the top of Fig. 2(a) indicates an apparent amplitude change of $\sim 1.4 \mathrm{~nm}_{\mathrm{p}-\mathrm{p}}$, as shown in the bottom of Fig. 2(a). When the laser-beam tracking system was switched on, this large error almost disappeared as shown in Fig. 2(b). The cross-section along the red line shown in the top of Fig. 2(b) shows an amplitude variation of only $\sim 0.2 \mathrm{~nm}$, as shown in the bottom of Fig. 2(b). It was also confirmed that the tracking system worked well even for faster imaging rates up to $10 \mathrm{fps}$.

\section{2-2. Combined Tip-scanning HS-AFM/TIRFM}

Figure 3(a) shows a schematic diagram of the OBD system for tip-scanning HS-AFM. An infrared (IR) laser of wavelength $980 \mathrm{~nm}$ is used for the OBD system instead of a red laser used in the standard HS-AFM system [51], because fluorescent dyes frequently used in fluorescence imaging emit fluorescence shorter than $800 \mathrm{~nm}$. The laser spot on the small cantilever can be monitored by the CCD camera through the objective lens and the dichroic mirror, which facilitates the alignment of the laser beam and the cantilever. The cantilever deflection is detected by the position-detection system consisting of the two-segmented Si PIN photodiode, amplifiers, and a signal conditioner with the bandwidth of $20 \mathrm{MHz}$. Other optical components are optimized for the IR wavelength, including the collimation lens, $\lambda / 4$ plate, polarization beam splitter, and the focusing lens placed at the front of the two-segmented PIN photodiode. A smaller spot size of the focused laser on the photodiode has a higher position-detection sensitivity for a given displacement of the laser spot. On the contrary, focusing into a small spot reduces the displacement of the laser spot caused by cantilever deflection. Therefore, when the gap size $(\Delta s)$ between the photodiode segments is not considered, the spot size $(D)$ has no effect on the sensitivity of cantilever deflection detection as far as the laser spot is within the effective area of the photodiode. However, taking the gap size into an account, the sensitivity to cantilever deflection increases with decreasing spot size by a factor of $\Delta s / D$. Nonetheless, the laser spot has to be set at an appropriate size so that 
centering the laser spot on the photodiode sensor is easily performed. When the spot size is very small, this centering adjustment becomes difficult. Considering these factors, we adjust the spot size in the range of $0.5 \sim 1 \mathrm{~mm}$ in diameter with the focusing lens.

Figures 3(b) shows the side view of the tip-scanning HS-AFM unit. The frame that supports the unit consists of the top and base parts. All components in the OBD detector including the mirror tilter are assembled in the top frame and hung from its roof to ensure mechanical stability. For coarse approach of the cantilever tip to the sample surface, a stepper motor is installed in the base part. The positions of the IR laser, the two-segmented photodiode, and the scanner with the cantilever can be adjusted with precise screws in the XZ-, YZ- and XY-planes, respectively. The whole assembly is supported by a tripod consisting of two adjustable screws and a screw connected to the revolving shaft of the stepper motor.

As optical microscopy to be combined with the tip-scanning HS-AFM, objective-lens type TIRFM was employed for single-molecule fluorescence imaging. The tip-scanning HS-AFM unit is simply mounted on a modified stage of the inverted optical microscope as shown in Fig 3(c). The stage of the inverted fluorescence microscope (hereafter referred to "optical stage") was modified to hang the objective lens directly from the optical stage, as was done for our first prototypic HS-AFM setup combined with an optical microscope [3]. This hanging was necessary for the following reason. An oil-immersion objective lens with a high NA has to be used for TIRFM and therefore the front face of the objective lens is in contact with a cover slip through immersion oil. In the standard configuration of inverted optical microscopes, the optical stage and the objective lens are mechanically separated; i.e., the objective lens is fixed in the revolver, while the optical stage is fixed on the top of the supporting frame. In this configuration, the objective lens and the optical stage vibrate nearly independently of each other, and therefore, vibrations of the large microscope body are easily transmitted to the cover slip mounted on the optical stage through the objective lens [52]. When the objective lens $(100 \times$, NA 1.49$)$ is hung directly from the optical stage, they vibrate together in phase, and hence, the vibration transmission is significantly suppressed.

Another modification of the optical stage was needed for precise assessment of the correlation between AFM and fluorescence images for the same sample. The field of view of our TIRFM setup is approximately $31 \times 31 \mu \mathrm{m}^{2}$. On the other hand, the lateral scan range of the tip-scanning HS-AFM system is currently limited to $800 \times 800 \mathrm{~nm}^{2}$. This large difference of the field of views complicates the correlation assessment. To fill this large gap, an additional scanner that moves the whole tip-scanning HS-AFM unit up to $15 \mu \mathrm{m}$ in the $\mathrm{X}$ - and Y-directions was implemented in the optical stage. For wide-area 
AFM imaging, this XY-scanner was used together with the Z-scanner of the tip-scanning HS-AFM system. Although its imaging rate is much lower (> $3 \mathrm{~min} /$ frame) than tip-scanning HS-AFM, the wide-area scanner enables precise positioning of the cantilever tip on a region of interest within a pre-acquired TIRFM image.

\section{2-3. Simultaneous HS-AFM/TIRFM Imaging}

Figure 4(a) shows a TIRFM image of rhodamine-labeled actin filaments that are partially biotinylated and immobilized on a cover slip sparsely covered with avidin molecules, while Figs. 4(c) and (d) show HS-AFM images of the same sample captured for narrow areas located within the region shown with the rectangle in Fig. 4(a). It seems impossible to correlate the HS-AFM images with the TIRFM image due to the crowded actin filaments and the poor resolution of the TIRFM image. Figure 4(b) shows a wide-area AFM image acquired as described above. By comparing the TRIFM and wide-area AFM images, we can easily find the positional correlation between the TIRFM image and the HS-AFM images captured for the narrow areas, as indicated with the small rectangles in the overlaid image between the TIRFM image and the wide-area AFM image, as shown in Fig. 4(e).

Chitinase $\mathrm{A}$ is an enzyme that degrades chitin microfibrils by hydrolysis and has been hypothesized to move processively and unidirectionally along the microfibrils [53]. This hypothesis was recently proven by HS-AFM imaging [22]. For fluorescence imaging, chitinase A was labeled with Cy3. Crystalline chitin microfibrils suspended in water were immobilized onto a glass cover slip by spin coating. Figure 5 shows successive images of chitinase A moving along a chitin microfibril, which were simultaneously captured by HS-AFM and TIRFM at an imaging rate of $3 \mathrm{fps}$. The HS-AFM images (upper panel in Fig. 5) show binding of a single chitinase A molecule to the chitin microfibril $(0.99 \mathrm{~s})$ and its afterwards movement on the microfibril towards the upper-right $(1.98 \mathrm{~s}$ and $2.64 \mathrm{~s})$. A fluorescent spot also appeared at $0.99 \mathrm{~s}$ in the TIRFM image (lower panel in Fig. 5). The fluorescent spot slightly moved with time toward the right side, although the movement is much less discernible, because the field of view in TIRFM is much wider than that in HS-AFM.

\section{2-4. Potential of Tip-scanning HS-AFM}

In addition to imaging, AFM has also been used as a force sensor to measure the strength of intra- and intermolecular bonds at the single molecule level and the elasticity of biological objects [53-56]. It is of note that the speed of stretching biological molecules is now approaching to that of molecular dynamics simulations [56]. Along 
with this progress, theoretical descriptions for bond rupture under a wide range of loading rates have recently been attempted [57]. The force measurements produce so-called the force-distance curves, only from which useful information has to be extracted. But the interpretation of the obtained curves is difficult unless the system under measurement is not well specified. Similarly, optical tweezers has been used in force and displacement measurements for single molecules and cells [58-60]. In the force measurements by these techniques, the objects under measurement cannot be visualized by AFM. It would be a breakthrough if we can visualize the objects under external force using HS-AFM. Tip-scanning HS-AFM can accommodate optical tweezers when it is combined with an optical microscope. Therefore, tip-scanning HS-AFM has a potential to visualize biological molecules under external force. It would provide a new opportunity to study the effect of applied force on the functional action of the molecules as well as detailed unfolding and refolding processes of the molecules.

Tip-enhanced fluorescence microscopy operates based on the significant enhancement of electric field of light in the vicinity of a metal or silicon tip that is illuminated with a laser beam [61]. It has resolution less than $10 \mathrm{~nm}$ [62]. However, this microscopy technique has not been of practical use, whereas other super-resolution fluorescence microscopy techniques are now being disseminated [39,63,64]. Tip-enhanced fluorescence microscopy can be combined with tip-scanning HS-AFM. When combined, both topological and fluorescence images with identical pixel positions would be acquired simultaneously at high spatiotemporal resolution.

\section{Wide-Area Scanner for Live Cell Imaging}

One of prospective applications of HS-AFM in biological science is high-resolution visualization of dynamic events occurring in live cells, such as endocytosis, exocytosis, and reorganization of cytoskeletons which occur autonomically or in response to external stimuli including mechanical and electrical stimuli, ligands for external membrane receptors and toxic substances.

Several techniques for fast, wide-area AFM imaging have been proposed [65-69], and this type of imaging has also been demonstrated for hard (or relatively hard) materials such as Si gratings [67-69] and some biological samples including dried collagen fibers [65], collagen fibers on bone trabecula [70] and live bacterial cells [66]. However, wide-area AFM imaging is difficult for soft biological samples such as live eukaryotic cells. To observe both bacterial and eukaryotic cells, we have developed a wide-area scanner with the maximum scan range of $\sim 46 \times 46 \mu \mathrm{m}^{2}$ [32]. To achieve precise, fast imaging using this scanner, we have also developed several control 
techniques for damping mechanical vibrations, expanding the frequency bandwidth, minimizing the nonlinear displacement of piezoactuators and the cross-talk between the $\mathrm{X}$ - and Y-displacements. These techniques are essential for a wide-area HS-AFM system but not necessarily required of the standard HS-AFM. In this section, we present the overviews of the design of the wide-area scanner and these control techniques, and finally describe application studies of wide-area HS-AFM to show it ability and potential.

\section{3-1. Design of Wide-Area Scanner}

Large displacements of a scanner in the X-and Y-directions can be achieved by the magnification of X-and Y-piezoactuators' displacements. For this magnification, we used the third-class leverage mechanism. Fig. 6(a) shows a schematic of the wide-area scanner. Its entire structure except the piezoactuators was made by monolithic fabrication of a stainless steel block. The X- and Y-piezoactuators are arranged symmetrically with respect to the central supporting base on which the Z-piezoactuator is attached. One end of the lever with a length of $25 \mathrm{~mm}$ is fixed to the frame, which works as a fulcrum. The point of effort, to which a long-stroke piezoactuator with a nominal unloaded displacement of $11.6 \mu \mathrm{m}$ at $100 \mathrm{~V}$ and the first resonant frequency of $69 \mathrm{kHz}$ is glued, is $5 \mathrm{~mm}$ distant from the fulcrum, i.e., the designed lever ratio is five. This design achieved the actual maximum scan ranges of $46.7 \mu \mathrm{m}$ for the X-direction and $45.7 \mu \mathrm{m}$ for the Y-direction. Figure 6(b) shows a photograph of the fully assembled scanner. The gap spaces in the scanner were filled with an elastomer consisting of polyurethane, which can passively damp low-frequency vibrations of the scanner.

\section{3-2 Control Techniques for Wide-Area Scanner}

\section{3-2-1. Vibration Damping}

For scanning in the $\mathrm{X}$-direction, a voltage signal with an isosceles triangle wave is usually applied to the X-scanner. However, high harmonic-frequency components are contained in the vertexes of the triangle wave, which easily excite the X-scanner with low resonant frequencies, resulting in generation of unwanted vibrations. The wide-area $\mathrm{X}$-scanner developed above has the first resonant frequency around $2 \mathrm{kHz}$ as shown in Fig. 7(a). The X-scanner generated serious vibrations (red line) as shown in Fig. 7(b), even though it was scanned by a triangle wave with the fundamental frequency of only $250 \mathrm{~Hz}$ (black line).

Instead of raster scanning, different two-dimensional scan trajectories, such as 
sinusoidal [67], spiral [71], cycloid [72] and Lissajous [73] curves, have been proposed. This is because in these cases the driving signals for the XY-scanner do not contain high harmonic components, and therefore, can prevent generation of unwanted vibrations. However, the procedures for producing the driving signals, reconstructing the image, and compensating for hysteresis effects of piezoactuators are complicated. Moreover, these scan trajectories would result in the exertion of lateral forces to the cantilever tip from various directions, and consequently the cantilever would be not only bent but also twisted by the tip-sample interaction. Therefore, the interaction force is hardly maintained constant. Here we used two methods and their combination to suppress the generation of unwanted vibrations; the inversion-based feed-forward damping [69] that is the same as that described for the mirror tilter [31], simple rounding of the vertexes of the triangle wave [74] and combination of these methods. Figure 6(c) shows the driving signal (black line) constructed by the inverse compensation method and the corresponding displacement of the X-scanner (red line). The construction of the driving signal was made using the frequency response shown in Fig. 7(a). The mechanical vibrations of the $\mathrm{X}$ scanner were significantly suppressed by this method as seen in Fig. $7(\mathrm{c})$.

The rounding of the vertexes of the triangle wave is simple and useful. There are several ways to generate rounded triangle waves with reduced higher harmonics. Rather than using low-pass filters, we generated a series of rounded triangle waves by removing higher harmonics terms from the Fourier series given in Eq. (1) and then calculating the inverse Fourier transform of the resulting Fourier series. Fig. 7(d) shows the rounded triangle wave constructed from harmonics up to the ninth order (black line) and the corresponding displacement of the X-scanner (red line). The displacement did not show noticeable vibrations. The nonlinear scan regions that only appear around the vertexes of the scan wave are about $10 \%$ of the full scan range, which would produce a nearly negligible effect on AFM images to be acquired.

However, even with these methods, the $\mathrm{X}$-scanner generated unwanted vibrations when scanned at $1 \mathrm{kHz}$. Then, we combined these methods. That is, the rounded triangle wave containing harmonics up to the ninth-order was filtered through the inversed transfer function obtained from the measured frequency response. As shown in Fig. 7(e), the $\mathrm{X}$-scanner was successfully driven at $1 \mathrm{kHz}$, without generating unwanted vibrations and without a significant deviation from linear trajectories in both trace and retrace regimes. Thus, the bandwidth of the X-scanner with the first resonant frequency of only $2 \mathrm{kHz}$ was significantly extended. 


\section{3-2-2. Compensation for Image Distortion}

The hysteresis nonlinearity of piezoactuators is not negligible for the case of wide-area AFM imaging. The positioning error due to the hysteresis is generally $10-15 \%$ of the full scan range, causing a significant distortion in acquired AFM images [75]. To compensate for the nonlinearity, the closed-loop control that is based on the actual displacements recorded by a displacement sensor is often used in commercial AFM systems. However, this control makes the scanner assembly complicated by the implementation of the displacement sensors. Moreover, the bandwidth of available high-precision displacement sensors is too low to be used in the high-speed scanner. The open-loop method that is based on feedforward compensation for the nonlinearity is rather simple [76]. For our wide-area scanner, we adopted the open-loop compensation method based on the pre-measured non-linear hysteresis curves of the $\mathrm{X}$ - and Y-scanners.

The nonlinearity of piezoactuators is independent of the displacement range. Therefore, we can construct a modified driving signal that can compensate for the nonlinearity, using the following procedures. The numerical data of the measured hysteresis curve of the displacement $d$ as a function of the applied voltage $V$ is first normalized by a given voltage $V_{0}$ and the corresponding displacement $d_{0}$. From this normalized curve $\bar{d}=\bar{d}(\bar{V})$, we obtain a curve of $\bar{V}=\bar{V}(\bar{d})$, where $\bar{d} \equiv d / d_{0}$ and $\bar{V} \equiv V / V_{0}$. This curve is fitted with a polynomial function. This polynomial function is used to calculate the driving signal that displaces the scanner up to a desired range. Fig. 8(a) shows the HS-AFM image of a square grating with a pitch of $10 \mu \mathrm{m}$ captured at 15 s/frame for a $33 \times 33 \mu \mathrm{m}^{2}$ area, without compensation for the nonlinearity. The image shows grating pitches with large variations of approximately $\pm 20 \%$ and $\pm 15 \%$ in the $\mathrm{X}$ - and the Y-directions, respectively. The use of the open-loop feedforward compensation method eliminated the nonlinearities, as shown in Fig. 8(b). The variations of the grating pitch are improved to be less than $\pm 1 \%$.

However, the image of the square grating is still distorted and appears rhombic, as shown in Fig. 8(c). This is due to displacement cross-talk (coupling) between the X-and Y-scanners. This cross-talk results from the fact that the free ends of the levers used in the displacement magnification mechanism cannot move straight. The coupling ratio was measured to be independent of the scan range. The ratio was 0.017 for the passive movement of the sample stage in the Y-direction when the X-scanner was displaced, while it was 0.023 for the passive movement of the sample stage in the X-direction when the Y-scanner was displaced. To eliminate this coupling, an appropriate fraction of the voltage applied to one piezoactuator (X- or Y-piezoactuator) was subtracted from the 
driving signal for the other piezoactuator. This simple method gave a nearly distortion-free image, as shown in Fig. 8(d).

\section{3-2-3. Z-scanner}

The Z-piezoactuator is one of the key devices that determine the feedback bandwidth [10,13]. Its resonant frequency should be high for HS-AFM but is restricted by the required maximum scan range. In general, the resonant frequency of a piezoactuator changes in inverse proportion to its maximum stroke. This conflict relationship has to be compromised especially for the observation of large-sized specimens. For the Z-scanner of the wide-area scanner, we chose a stack piezoactuator with a displacement of $\sim 2.5 \mu \mathrm{m}$ at $100 \mathrm{~V}$ and the first resonant frequency of $222 \mathrm{kHz}$ in free oscillation. This piezoactuator was glued on the top of the central supporting base to be moved in the $\mathrm{X}$ - and Y-directions. The same type of piezoactuator (Z'-piezoactuator) was also glued to the bottom of the supporting base to counteract the impulsive force exerted to the supporting base by quick displacement of the Z-piezoactuator [3,10]. Moreover, the mechanical resonances of both $Z$ - and Z'-piezoactuators were suppressed by an active damping method based on the quality factor control technique that uses an LRC circuit, as a mock scanner, having a frequency response similar to those of the Z- and Z'-piezoactuators attached to the supporting base [10].

\section{3-3. Cantilever Tip for Cell Imaging}

The small cantilevers (BL-AC7DS-KU2 and BL-AC10DS-A2, Olympus, Tokyo) that we have been being used for HS-AFM have a bird beak-like tip end. But the tip is not sharp enough to gain high-resolution images. To have a stylus-shaped sharp tip, we deposit amorphous carbon on the very end of the original tip by electron beam deposition using the spot mode of scanning electron microscopy (SEM), and then sharpen the grown tip by RF-plasma etching under argon environment. The tip end radius results in 3-5 $\mathrm{nm}$. The length of the carbon tip used for imaging of biological molecules is usually $\sim 1 \mu \mathrm{m}$. Since the growth rate of carbon tip is approximately 600 $\mathrm{nm} / \mathrm{min}$, the focused electron beam is usually irradiated for 1-2 min. However, the tip with length of $\sim 1 \mu \mathrm{m}$ is unsuited for the observation of live cells of higher than a few $\mu \mathrm{m}$ as the cantilever itself would directly contact with the cell surface. To obtain a longer tip, the electron beam deposition for 1 min was repeated 5-7 times [77]. For each time of deposition, the focus position of the electron beam was readjusted to compensate for mechanical drift of the SEM. This procedure produces a tip with length 
of $\sim 3 \mu \mathrm{m}$. Its treatment by RF-plasma etching was briefly performed (for $\sim 10 \mathrm{~s}$ ) to prevent shortening of the tip, resulting in the tip end radius of $5-8 \mathrm{~nm}$.

\section{3-4. Positioning of Tip on Cell Surface}

The maximum scan range of the wide-area scanner, $\sim 46 \times 46 \mu \mathrm{m}^{2}$, is usually still too small to observe a whole eukaryote cell. A central region of a eukaryotic cell without underlying stiff structures is easily deformed by the loading force from the cantilever tip and therefore hard to be imaged. Therefore, we needed to position the cantilever tip onto flat peripheral regions of a cell supported by the stiff substrate. In our HS-AFM setup, the small cantilever can be monitored using a CCD camera, allowing us to align the positions of the cantilever, the sample stage and the laser spot focused onto the cantilever. However, the cells on the sample stage cannot be observed by this optical system, and therefore, tip positioning at a desired location of the cell sample cannot be conducted. To solve this problem, we added a simple fluorescent microscopy system to the standard HS-AFM setup, as shown in Fig. 9(a) [47,77]; the excitation lamp, dichroic filters and the CCD camera are installed in the bottom part of the HS-AFM setup. By using this system and cells transfected with green fluorescent protein (GFP), the cantilever tip can be positioned at an arbitrary region of the cell sample (Fig. 9(b)).

\section{3-5. Live Cell Imaging}

First, the wide-area scanner was used for the observation of the bacteriolysis process of rod-shaped Bacillus (B.) subtilis subjected to lysozyme. B. subtilis is susceptible to antibacterial action of lysozyme that hydrolyzes 1,4-beta-linkages between $N$-acetyl glucosamine and $N$-acetyl muramic acid, leading to degradation of peptidoglycan on the cell wall [78]. We monitored morphological changes of the cell surface caused by this degradation to demonstrate the capability of wide-area HS-AFM [32]. The bacterial cells were immobilized on a glass stage coated with poly-L-lysine $(0.1 \mathrm{mg} / \mathrm{ml})$ and then imaged in a culture medium (Fig. 10(a)). Fig. 10 (b) shows clipped images of the bacterium before and after the addition of lysozyme, captured at $20 \mathrm{~s} /$ frame $(256 \times 256$ pixels). At $t=0 \mathrm{~s}$, the bacterial cell surface appeared smooth. At $t=240 \mathrm{~s}$, lysozyme was added to the observation solution at the final concentration of $80 \mu \mathrm{M}$. After $t=320$ $\mathrm{s}$, the surface morphology gradually varied and wrinkle structures perpendicular to the long cell axis appeared, while the whole cell gradually swelled $(t=1060 \mathrm{~s}, 1460 \mathrm{~s}$ and $1580 \mathrm{~s})$. Finally, at $t=1720 \mathrm{~s}$, the cell burst presumably due to an excessive osmotic pressure from the inside of the cell.

Next, we observed eukaryotic COS-7 and Hela cells that are much softer than 
bacterial cells [77]. Here, we only show the results with COS-7. The COS-7 cells were transfected with monomeric enhanced GFP (mEGFP). The cantilever tip was positioned at a peripheral region of one of the cells under the simple fluorescence microscope (Fig. 11 (a)). The membrane dynamics and its activation induced by insulin were observed in real time. Figs. 11 (b) and (c) show topographic images captured for the peripheral region of the same COS-7 cell before and after the addition of insulin, respectively. Figs. 11(d) and (e) show HS-AFM images taken at the indicated times (d) before and (e) after the insulin application. These images are overlaid with the corresponding images captured at $\mathrm{t}=0 \mathrm{~s}$ (i.e., images (b) and (c)). The areas shown in green and magenta indicate newly appeared and disappeared structures in the entire imaged region, respectively, while the filled and open white arrows indicate newly appeared and disappeared structures at the leading edge of the cell, respectively. It can be seen that the membrane ruffling at the leading edge of the cell is activated in response to insulin application.

We also observed morphological changes of live neurons. Hippocampal neurons from newborn rats were planted at low density on a top layer of glia cells placed on a glass stage and cultured for 9 days [79]. Under the fluorescence microscope, the AFM tip was positioned near thin dendrites of the neuron transfected by mEGFP, as shown in Fig. 12(a). As seen in Fig. 12(b), rapid filopodia extension and retraction were observed. Furthermore, a thin and sheet-like ruffling structure on top of glia cells around dendritic shafts was also observed (data not shown). This structure would be difficult to image under an optical microscope due to its small width $(150-500 \mathrm{~nm})$ and thickness $(\sim 70$ $\mathrm{nm}$ ), which are beyond the resolution of conventional optical microscopes.

\section{Summary}

The maturity of HS-AFM has enabled us to directly visualize various dynamic processes occurring in biological molecules in real time, and consequently, gain more detailed and deeper insights into their functional mechanisms. Combination of HS-AFM with other powerful techniques that can provide complimentary information should make HS-AFM more powerful and versatile in biological science. In this review, we first described very recent technical developments of tip-scanning HS-AFM that can be easily combined with fluorescence microscopy. This combination allows us to acquire the information of both conformational dynamics and chemical states of individual protein molecules. This will open an opportunity to precisely identify conformational changes of a protein molecule triggered by, for example, nucleotide binding, hydrolysis, and product release. Moreover, the identification of molecular species by fluorescence 
microscopy during HS-AFM imaging makes it easier to interpret AFM images of complex systems consisting of several species of molecules. However, for this task, the combined system we developed is not powerful enough, as the spatial resolution differs largely between the two microscopies. And yet, this will soon be resolved because in principle the tip-scanning HS-AFM can be combined with confocal microscopy and super-resolution fluorescence microscopy including stimulated emission depletion (STED) microscopy [39], stochastic optical reconstruction microscopy (STORM) [61], and photo-activated localization microscopy (PALM) [60]. Such ultra-hybrid microscopy systems will bring a major impact on single-molecule biophysics of isolated biological molecules and a less impact on cell biology as briefly described below. Optical tweezers can also be incorporated to tip-scanning HS-AFM, which will open a new opportunity to visualize proteins molecules under external force. Tip-scanning HS-AFM can also serve for non-biological or industrial applications, as it can accommodate large and heavy specimens, such as semiconductor wafers.

The wide-area scanner is also an important technical breakthrough that extends the application rage of HS-AFM from single molecules to live cells. We described the mechanical design of the wide-area scanner as well as several control techniques to suppress unwanted vibrations, nonlinear displacement of piezoactuators and cross-talk between two lateral scan axes. Now, more complex systems including live cells and organisms have become targets of high-speed AFM. Although we have not yet completed, the implementation of the wide-area scanner in the tip-scanning HS-AFM system combined with an optical microscope will largely expand the capability and functionality of HS-AFM. As AFM only visualizes a surface, its use with fluorescence microscopy must be also useful for studying dynamic molecular events occurring on the surfaces (not in interiors) of live cells or isolated organelles. However, its targets are limited to small and hence relatively rigid structures like neuronal spines, small mitochondria and nuclei. Nevertheless, in-situ visualization of dynamic molecular events occurring on these higher-order structures will bring impact on cell biology, as dynamic molecular phenomena occurring there are mostly unclear.

\section{Acknowledgements}

This work was supported by the CREST program of the Japan Science and Technology Agency (JST) (to T.A.), the JST program on Development of Systems and Technology for Advanced Measurement and Analysis (to T.A.), Grant-in-Aids for Basic Research (\#24227005 to T.A., and \#23115008, \#24241048, \#26104514 and \#26102515 to T.U.), Grant-in-Aid for Research on Innovative Area (\#26119003 to T.A.), Human Frontier 
Research Program (to T.A.) and Grant-in-Aid for Research Fellows (to S.F.) from the Japan Society for the Promotion of Science. 


\section{References}

[1] G. Binnig, C.F. Quate, C. Gerber, Atomic force microscope, Phys. Rev. Lett. 56 (1986) 930-933. DOI: 10.1103/PhysRevLett.56.930

[2] J.H. Kindt, G.E. Fantner, J.A. Cutroni, P.K. Hansma, Rigid design of fast scanning probe microscopes using finite element analysis, Ultramicroscopy 100 (2004) 259-265. DOI:10.1016/j.ultramic.2003.11.009

[3] T. Ando, N. Kodera, E. Takai, D. Maruyama, K. Saito, A. Toda, A high-speed atomic force microscope for studying biological macromolecules, Proc. Natl. Acad. Sci. U. S. A. 98 (2001) 12468-12472. DOI: 10.1073/pnas.211400898

[4] T. Ando, T. Uchihashi, N. Kodera, A. Miyagi, R. Nakakita, H. Yamashita, K. Matada, High-speed AFM for studying the dynamic behavior of protein molecules at work, e-J. Surf. Sci. Nanotechnol. 3 (2005) 384-392. DOI: 10.1380/ejssnt.2005.384

[5] M.B. Viani, T.E. Schaffer, A. Chand, M. Rief, H.E. Gaub, P.K. Hansma, Small cantilevers for force spectroscopy of single molecules, J. Appl. Phys. 86 (1999) 2258-2262. DOI: 10.1063/1.371039

[6] D.A. Walters, J.P. Cleveland, N.H. Thomson, P.K. Hansma, M.A. Wendman, G. Gurley, V. Elings, Short cantilevers for atomic force microscopy, Rev. Sci. Instrum. 67 (1996) 3583-3590. DOI: $\underline{\text { 10.1063/1.1147177 }}$

[7] M. Kitazawa, K. Shiotani, A. Toda, Batch fabrication of sharpened silicon nitride tips, Jpn. J. Appl. Phys. 42 (2003) 4844-4847. DOI: 10.1143/JJAP.42.4844

[8] J. Kokavecz, Z. Toth, Z.L. Horvath, P. Heszler, A. Mechler, Novel amplitude and frequency demodulation algorithm for a virtual dynamic atomic force microscope, Nanotechnology 17 (2006) S173-S177. DOI: 10.1088/0957-4484/17/7/S12

[9] M.B. Viani, L. I. Pietrasanta, J.B. Thompson, A. Chand, I.C. Gebeshuber, J.H. Kindt, M. Richter, H.G. Hansma, P.K. Hansma, Probing protein-protein interactions in real time, Nat. Struct. Biol. 7 (2000) 644-647. DOI: 10.1038/77936

[10] N. Kodera, H. Yamashita, T. Ando, Active damping of the scanner for high-speed atomic force microscopy, Rev. Sci. Instrum. 76 (2005) 053708. DOI: $10.1063 / 1.1903123$

[11] N. Kodera, M. Sakashita, T. Ando, Dynamic proportional-integral-differential controller for high-speed atomic force microscopy, Rev. Sci. Instrum. 77 (2006) 083704. DOI: $10.1063 / 1.2336113$

[12] T. Uchihashi, T. Ando, H. Yamashita, Fast phase imaging in liquids using a rapid scan atomic force microscope, Appl. Phys. Lett. 89 (2006) 213112. DOI: $10.1063 / 1.2387963$ 
[13] T. Ando, T. Uchihashi, T. Fukuma, High-speed atomic force microscopy for nano-visualization of dynamic biomolecular processes, Prog. Surf. Sci. 83 (2008) 337-437. DOI: 10.1016/j.progsurf.2008.09.001

[14] M. Shibata, T. Uchihashi, H. Yamashita, H. Kandori, T. Ando, Structural changes in bacteriorhodopsin in response to alternate illumination observed by high-speed atomic force microscopy, Angew. Chem. Int. Ed. 50 (2011) 4410-4413. DOI: 10.1002/anie.201007544

[15] M. Shibata, H. Yamashita, T. Uchihashi, H. Kandori, T. Ando, High-speed atomic force microscopy shows dynamic molecular processes in photoactivated bacteriorhodopsin, Nat. Nanotechnol. 5 (2010) 208-212. DOI: 10.1038/nnano.2010.7

[16] H. Yamashita, K. Inoue, M. Shibata, T. Uchihashi, J. Sasaki, H. Kandori, T. Ando, Role of trimer-trimer interaction of bacteriorhodopsin studied by optical spectroscopy and high-speed atomic force microscopy, J. Struct. Biol. 184 (2013) 2-11. DOI: 10.1016/j.jsb.2013.02.011.

[17] T. Uchihashi, R. Iino, T. Ando, H. Noji, High-speed atomic force microscopy reveals rotary catalysis of rotorless F(1)-ATPase, Science 333 (2011) 755-758. OI: 10.1126/science. 1205510

[18] N. Kodera, D. Yamamoto, R. Ishikawa, T. Ando, Video imaging of walking myosin V by high-speed atomic force microscopy, Nature 468 (2010) 72-76. DOI: 10.1038/nature09450

[19] A. Nakamura, H. Watanabe, T. Ishida, T. Uchihashi, M. Wada, T. Ando, K. Igarashi, M. Samejima, Trade-off between processivity and hydrolytic velocity of cellobiohydrolases at the surface of crystalline cellulose, J. Am. Chem. Soc. 136 (2014) 4584-4592. DOI: $10.1021 / \mathrm{ja} 4119994$

[20] K. Igarashi, T. Uchihashi, A. Koivula, M. Wada, S. Kimura, T. Okamoto, M. Penttilä, T. Ando, M. Samejima, Traffic jams reduce hydrolytic efficiency of cellulase on cellulose surface, Science 333 (2011) 1279-1282. DOI: 10.1126/science.1208386

[21] K. Igarashi, A. Koivula, M. Wada, S. Kimura, M. Penttilä, M. Samejima, Highspeed atomic force microscopy visualizes processive movement of trichoderma reesei cellobiohydrolase I on crystalline cellulose, J. Biol. Chem. 284 (2009) 36186-36190. DOI: $10.1074 /$ jbc.M109.034611

[22] K. Igarashi, T. Uchihashi, T. Uchiyama, H. Sugimoto, M. Wada, K. Suzuki, S. Sakuda, T. Ando, T. Watanabe, M. Samejima, Two-way traffic of glycoside hydrolase family 18 processive chitinases on crystalline chitin, Nat. Commun. 5 (2014) 3975. DOI: $10.1038 /$ ncomms4975

[23] A. Laisne, M. Ewald, T. Ando, E. Lesniewska, D. Pompon, Self-assembly 
properties and dynamics of synthetic proteo-nucleic building blocks in solution and on surfaces, Bioconj. Chem. 22 (2011) 1824-1834. DOI: 10.1021/bc2002264

[24] D. Yamamoto, N. Nagura, S. Omote, M. Taniguchi, T. Ando, Streptavidin 2D crystal substrates for visualizing biomolecular processes by atomic force microscopy, Biophys. J. 97 (2009) 2358-2367. DOI: 10.1016/j.bpj.2009.07.046

[25] D. Yamamoto, T. Uchihashi, N. Kodera, T. Ando, Anisotropic diffusion of point defects in two-dimensional crystal of streptavidin observed by high-speed atomic force microscopy, Nanotechnology 19 (2008) 384009.

[26] A. Colom, I. Casuso, T. Boudier, S. Scheuring, High-speed atomic force microscopy: cooperative adhesion and dynamic equilibrium of junctional microdomain membrane proteins, J. Mol. Biol. 423 (2012) 249-256. DOI: 10.1088/0957-4484/19/38/384009

[27] S. Ishino, T. Yamagami, M. Kitamura, N. Kodera, T. Mori, S. Sugiyama, T. Ando, N. Goda, T. Tenno, H. Hiroaki, Y. Ishino, Multiple interactions of the intrinsically disordered region between the helicase and nuclease domains of the archaeal Hef protein, J. Biol. Chem. 289 (2014) 21627-21639. DOI: 10.1074/jbc.M114.554998

[28] A. Miyagi, Y. Tsunaka, T. Uchihashi, K. Mayanagi, S. Hirose, K. Morikawa, T. Ando, Visualization of intrinsically disordered regions of proteins by high-speed atomic force microscopy, Chem. Phys. Chem. 9 (2008) 1859-1866. DOI: 10.1002/cphc. 200800210

[29] T. Ando, T. Uchihashi, S. Scheuring, Filming biomolecular processes by high-speed atomic force microscopy, Chem. Rev. 114 (2014) 3120-3188. DOI: $10.1021 /$ cr4003837

[30] T. Ando, T. Uchihashi, N. Kodera, High-speed AFM and applications to biomolecular systems, Annu. Rev. Biophys. $42 \quad$ (2013) 393-414. DOI: 10.1146/annurev-biophys-083012-130324

[31] S. Fukuda, T. Uchihashi, R. Iino, Y. Okazaki, M. Yoshida, K. Igarashi, T. Ando, High-speed atomic force microscope combined with single-molecule fluorescence microscope, Rev. Sci. Instrum. 84 (2013) 073706. DOI: 10.1063/1.4813280

[32] H. Watanabe, T. Uchihashi, T. Kobashi, M. Shibata, J. Nishiyama, R. Yasuda, T. Ando, Wide-area scanner for high-speed atomic force microscopy, Rev. Sci. Instrum. 84 (2013) 053702. DOI: 10.1063/1.4803449

[33] B. Huang, M. Bates, X. Zhuang, Super-resolution fluorescence microscopy, Annu. Rev. Biochem. 78 (2009) 993-1016. DOI: 10.1146/annurev.biochem.77.061906.092014 [34] H. Park, E. Toprak, P.R. Selvin, Single-molecule fluorescence to study molecular motors, Q. Rev. Biophys. 40 (2007) 87-111. DOI: 10.1017/S0033583507004611 
[35] C. Joo, H. Balci, Y. Ishitsuka, C. Buranachai, T. Ha, Advances in single-molecule fluorescence methods for molecular biology, Annu. Rev. Biochem. 77 (2008) 51-76. DOI: 10.1146/annurev.biochem.77.070606.101543

[36] Y. Sako, Imaging single molecules in living cells for systems biology, Mol. Syst. Biol. 2 (2006) 56. DOI: 10.1038/msb4100100

[37] A. Ishijima, H. Kojima, T. Funatsu, M. Tokunaga, H. Higuchi, H. Tanaka, T. Yanagida, Simultaneous observation of individual ATPase and mechanical events by a single myosin molecule during interaction with actin, Cell 92 (1998) 161-171. DOI: 10.1016/S0092-8674(00)80911-3

[38] M. Tokunaga, K. Kitamura, K. Saito, A.H. Iwane, T. Yanagida, Single molecule imaging of fluorophores and enzymatic reactions achieved by objective-type total internal reflection fluorescence microscopy, Biochem. Biophys. Res. Commun. 235 (1997) 47-53. DOI: 10.1006/bbrc.1997.6732

[39] K.I. Willig, S.O. Rizzoli, V. Westphal, R. Jahn, S.W. Hell, STED microscopy reveals that synaptotagmin remains clustered after synaptic vesicle exocytosis, Nature 440 (2006) 935-939. DOI: 10.1038/nature04592

[40] J. Buckers, D. Wildanger, G. Vicidomini, L. Kastrup, S.W. Hell, Simultaneous multi-lifetime multi-color STED imaging for colocalization analyses, Opt. Express 19 (2011) 3130-3143. DOI: 10.1364/OE.19.003130

[41] M. Bates, B. Huang, G.T. Dempsey, X.W. Zhuang, Multicolor super-resolution imaging with photo-switchable fluorescent probes, Science 317 (2007) 1749-1753. DOI: $10.1126 /$ science. 1146598

[42] R. Kassies, K.O. Van der Werf, A. Lenferink, C.N. Hunter, J.D. Olsen, V. Subramaniam, C. Otto, Combined AFM and confocal fluorescence microscope for applications in bio-nanotechnology, J. Microsc.-Oxford 217 (2005) 109-116. DOI: 10.1111/j.0022-2720.2005.01428.x

[43] S. Nishida, Y. Funabashi, A. Ikai, Combination of AFM with an objective-type total internal reflection fluorescence microscope (TIRFM) for nanomanipulation of single cells, Ultramicroscopy 91 (2002) 269-274. DOI: 10.1016/S0304-3991(02)00108-0

[44] A.B. Mathur, G.A. Truskey, W.M. Reichert, Atomic force and total internal reflection fluorescence microscopy for the study of force transmission in endothelial cells, Biophys. J. 78 (2000) 1725-1735. DOI: 10.1016/S0006-3495(00)76724-5

[45] H. Nakajima, Y. Kunioka, K. Nakano, K. Shimizu, M. Seto, T. Ando, Scanning force microscopy of the interaction events between a single molecule of heavy meromyosin and actin, Biochem. Biophys. Res. Commun. 234 (1997) 178-182. DOI: 
10.1006/bbrc.1997.6612

[46] Y. Suzuki, N. Sakai, A. Yoshida, Y. Uekusa, A. Yagi, Y. Imaoka, S. Ito, K. Karaki, K. Takeyasu, High-speed atomic force microscopy combined with inverted optical microscopy for studying cellular events, Sci. Rep. 3 (2013) 2131. DOI: 10.1038/srep02131

[47] A. Colom, I. Casuso, F. Rico, S. Scheuring, A hybrid high-speed atomic force-optical microscope for visualizing single membrane proteins on eukaryotic cells, Nat. Commun. 4 (2013) 2155.

[48] K.O. Vanderwerf, C.A.J. Putman, B.G. Degrooth, F.B. Segerink, E.H. Schipper, N.F. Vanhulst, J. Greve, Compact stand-alone atomic-force microscope, Rev. Sci. Instrum. 64 (1993) 2892-2897. DOI: 10.1038/ncomms3155

[49] K. Nakano, A novel low profile atomic force microscope compatible with optical microscopes, Rev. Sci. Instrum. 69 (1998) 1406-1409. DOI: 10.1063/1.1148774

[50] G. Schitter, A. Stemmer, Identification and open-loop tracking control of a piezoelectric tube, scanner for high-speed scanning-probe microscopy, IEEE Trans. Control Syst. Technol. 12 (2004) 449-454. DOI: 10.1109/TCST.2004.824290

[51] T. Uchihashi, N. Kodera, T. Ando, Guide to video recording of structure dynamics and dynamic processes of proteins by high-speed atomic force microscopy, Nat. Protoc. 7 (2012) 1193-1206. DOI: 10.1038/nprot.2012.047

[52] H. Gumpp, S.W. Stahl, M. Strackharn, E.M. Puchner, H.E. Gaub, Ultrastable combined atomic force and total internal fluorescence microscope, Rev. Sci. Instrum. 80 (2009) 063704. DOI: 10.1063/1.3148224

[53] T. Uchiyama, F. Katouno, N. Nikaidou, T. Nonaka, J. Sugiyama, T. Watanabe, Roles of the exposed aromatic residues in crystalline chitin hydrolysis by chitinase a from Serratia marcescens, J. Biol. Chem. 276 (2001) 41343-41349. DOI:

\subsection{4/jbc.M103610200}

[54] M. Rief, F. Oesterhelt, B. Heymann, H.E. Gaub, Single molecule force spectroscopy on polysaccharides by atomic force microscopy, Science 275 (1997) 1295-1297. DOI: 10.1126/science.275.5304.1295

[55] M. Rief, M. Gautel, F. Oesterhelt, J.M. Fernandez, H.E. Gaub, Reversible unfolding of individual titin immunoglobulin domains by AFM, Science 276 (1997) 1109-1112. DOI: $10.1126 /$ science.276.5315.1109

[56] F. Rico, L. Gonzalez, I. Casuso, M. Puig-Vidal, S. Sheuring, High-speed force spectroscopy unfolds titin at the velocity of molecular dynamics simulations, Science 342 (2013) 741-743. DOI: 10.1126/science.1239764

[57] J. T. Bullerjarn, S. Sturm and K. Kroy, Theory of rapid force spectroscopy, Nat. 
Commun. 5 (2014) 4463. DOI: 10.1038/ncomms5463

[58] H. Zhang, K.K. Liu, Optical tweezers for single cells, J. Roy. Soc. Int. 5 (2008) 671-690. DOI: 10.1098/rsif.2008.0052

[59] K.C. Neuman, A. Nagy, Single-molecule force spectroscopy: optical tweezers, magnetic tweezers and atomic force microscopy, Nat. Methods 5 (2008) 491-505. DOI: 10.1038/nmeth. 1218

[60] R.J. Davenport, G.J.L. Wuite, R. Landick, C. Bustamante, Single-molecule study of transcriptional pausing and arrest by E-coli RNA polymerase, Science 287 (2000) 2497-2500. DOI: 10.1126/science.287.5462.2497

[61] N. Hayazawa, Y. Inouye, S. Kawata, Evanescent field excitation and measurement of dye fluorescence in a metallic probe near-field scanning optical microscope, J. Microsc.-Oxford 194 (1999) 472-476. DOI: 10.1046/j.1365-2818.1999.00563.x

[62] J.M. Gerton, L.A. Wade, G.A. Lessard, Z. Ma, S.R. Quake, Tip-enhanced fluorescence microscopy at 10 nanometer resolution, Phys. Rev. Lett. 93 (2004) 180801. DOI: 10.1103/PhysRevLett.93.180801

[63] E. Betzig, G.H. Patterson, R. Sougrat, O.W. Lindwasser, S. Olenych, J.S. Bonifacino, M.W. Davidson, J. Lippincott-Schwartz, H.F. Hess, Imaging intracellular fluorescent proteins at nanometer resolution, Science 313 (2006) 1642-1645. DOI: $10.1126 /$ science.1127344

[64] M.J. Rust, M. Bates, X.W. Zhuang, Sub-diffraction-limit imaging by stochastic optical reconstruction microscopy (STORM), Nat. Methods 3 (2006) 793-795. DOI: 10.1038/nmeth929

[65] C. Braunsmann, T.E. Schaffer, High-speed atomic force microscopy for large scan sizes using small cantilevers, Nanotechnology 21 (2010) 225705. DOI: 10.1088/0957-4484/21/22/225705

[66] G.E. Fantner, R.J. Barbero, D.S. Gray, A.M. Belcher, Kinetics of antimicrobial peptide activity measured on individual bacterial cells using high-speed atomic force microscopy, Nat. Nanotechnol. 5 (2010) 280-285. DOI: 10.1038/nnano.2010.29

[67] A.J. Fleming, B.J. Kenton, K.K. Leang, Bridging the gap between conventional and video-speed scanning probe microscopes, Ultramicroscopy 110 (2010) 1205-1214. [68] I. Choi, Y. Kim, J.H. Kim, Y.I. Yang, J. Lee, S. Lee, S. Hong, J. Yi, Fast image scanning method in liquid-AFM without image distortion, Nanotechnology 19 (2008) 445701. DOI: 10.1016/j.ultramic.2010.04.016

[69] G. Schitter, K.J. Astrom, B.E. DeMartini, P.J. Thurner, K.L. Turner, P.K. Hansma, Design and modeling of a high-speed AFM-scanner, IEEE Trans. Control Syst. Technol. 15 (2007) 906-915. DOI: 10.1109/TCST.2007.902953 
[70] G. Schitter, P.J. Thurner, P.K. Hansma, Design and input-shaping control of a novel scanner for high-speed atomic force microscopy, Mechatronics 18 (2008) 282-288. DOI: 0.1016/j.mechatronics.2008.02.007

[71] I.A. Mahmood, S.O.R. Moheimani, Fast spiral-scan atomic force microscopy, Nanotechnology 20 (2009) 365503. DOI: 10.1088/0957-4484/20/36/365503

[72] Y.K. Yong, S.O.R. Moheimani, I.R. Petersen, High-speed cycloid-scan atomic force microscopy, Nanotechnology 21 (2010) 365503. DOI: 10.1088/0957-4484/21/36/365503

[73] A. Bazaei, Y.K. Yong, S.O.R. Moheimani, High-speed Lissajous-scan atomic force microscopy: Scan pattern planning and control design issues, Rev. Sci. Instrum. 83 (2012) 063701. DOI: 10.1063/1.4725525.

[74] K.K. Leang, A.J. Fleming, High-Speed Serial-Kinematic Spm Scanner: Design and drive considerations, Asian J. Control 11 (2009) 144-153. DOI: 10.1002/asjc.090

[75] P. Ge, M. Jouaneh, Tracking control of a piezoceramic actuator, IEEE Trans. Control Syst. Technol. 4 (1996) 209-216. DOI: 10.1109/87.491195

[76] B. Mokaberi, A.A.G. Requicha, Compensation of scanner creep and hysteresis for AFM nanomanipulation, IEEE Trans. Autom. Sci. Eng. 5 (2008) 197-206. DOI: 10.1109/TASE.2007.895008

[77] M. Shibata, T. Uchihashi, T. Ando, R. Yasuda, Long-tip high-speed atomic force microscopy for nanometer-scale imaging in live cells, Sci. Rep. 5 (2015) 8724. DOI: 10.1038/srep08724

[78] J.M. Ghuysen, Use of bacteriolytic enzymes in determination of wall structure and their role in cell metabolism, Bacteriol. Rev. 32 (1968) 40.

[79] T. Fath, Y.D. Ke, P. Gunning, J. Gotz, L.M. Ittner, Primary support cultures of hippocampal and substantia nigra neurons, Nat. Protoc. 4 (2009) 78-85. DOI: 10.1038/nprot.2008.199 


\section{Figure Captions}

Fig. 1 Dichroic mirror tilter for laser-beam tracking of lateral cantilever motion and its vibration damping. (a) Schematic showing the working principle of laser-beam tracking by mirror-tilting method. (b), (c) Perspective (b) and side (c) views of the two-dimensional mirror tilter. (d) Tilting motion of X-tilter (red line) driven by $1 \mathrm{kHz}$ isosceles triangle wave signal (black line). (c) Tilting motion of X-tilter (red line) driven by $1 \mathrm{kHz}$ isosceles triangle signal filtered through inverse compensation (black line).

Fig. 2 Effect of laser-beam tracking of lateral cantilever motion on the OBD detection of cantilever oscillation amplitude. (a) Amplitude image captured at $1 \mathrm{fps}$ for $650 \times 650$ $\mathrm{nm}^{2}(256 \times 256$ pixels $)$ without laser-beam tracking. The bottom shows a height profile of the image along the diagonal red line. (b) Amplitude image captured at $1 \mathrm{fps}$ for 650 $\times 650 \mathrm{~nm}^{2}(256 \times 256$ pixels $)$ with laser-beam tracking. The bottom shows a height profile of the image along the diagonal red line. For these imaging experiments, the cantilever tip is not in contact with the substrate surface.

Fig. 3 Schematics and photograph showing the structure of tip-scan HS-AFM unit. (a) Configuration of OBD detector with dichroic mirror tilter installed in the HS-AFM unit. (b) Whole structure of the tip-scan HS-AFM unit. The numbers attached to respective parts correspond to the numbers shown in (a). (c) A photograph of the tip-scan HS-AFM unit mounted on the optical stage in which the wide-area XY-scanner is installed.

Fig. 4 Positional correlation between TIRFM, AFM, and HS-AFM images of partially biotinylated and fully rhodamine-labeled actin filaments placed on a sparsely avidine-coated cover slip. (a) TIRFM image. The white rectangle indicates the region for which (b) a wide-area AFM image was taken at $10 \mathrm{~min} /$ frame. (c) HS-AFM image captured at an imaging rate of $8.3 \mathrm{fps}$ with $100 \times 45$ pixels. The position of cantilever tip was set by reference to the TIRFM image. (d) HS-AFM image captured at an imaging rate of $2 \mathrm{fps}$ with $100 \times 100$ pixels. The position of cantilever tip was set by reference to the TIRFM image. (e) The overlaid TERM and AFM image. The imaged areas of (c) and (d) are those shown by the respective white rectangles. 
Fig. 5 Simultaneous HS-AFM/TIRFM imaging of proteins in action. (a) Cy3-labeled chitinase A on a chitin microfibril. The HS-AFM images (upper panel) captured at $3 \mathrm{fps}$ with $150 \times 35$ pixels show a single chitinase A molecule moving towards the top-right (arrows). The TIRFM images (lower panel) with $40 \times 15$ pixels also show the same moving chitinase A molecule (arrows).

Fig. 6 Design of wide-area scanner. (a) Structure of a newly designed wide-area scanner for sample stage-scanning high-speed AFM. (b) A photograph of the wide-area scanner.

Fig. 7 Vibration damping of the wide-area X-scanner. (a) Frequency spectra of mechanical response of the X-scanner (red line, amplitude; blue line, phase). (b) $256 \mathrm{~Hz}$ driving signal of non-modified triangular wave (black line) and corresponding displacement of the X-scanner (red line). (c) $256 \mathrm{~Hz}$ driving signal of triangle wave modified by the inversed compensation method (black line) and corresponding displacement of the X-scanner (red line). (d) $85 \mathrm{~Hz}$ driving signal of rounded triangle wave containing harmonics up to the 9the order (black line) and corresponding displacement of the $\mathrm{X}$-scanner. (e) $1 \mathrm{kHz}$ driving signal of triangle wave treated by both vertex rounding and inverse compensation (black line) and corresponding displacement of the X-scanner (red line).

Fig. 8 Compensation for nonlinearity of piezoactuators and cross-talk between $\mathrm{X}$ - and Y-scanners. (a) AFM image of a square-shaped grating sample with a pitch of $10 \mu \mathrm{m}$ obtained without compensation for nonlinearity (imaging rate, 7 s/frame; pixels, $256 \times$ 256). (b) AFM image of the same grating sample obtained by compensation for nonlinearity (imaging rate, $7 \mathrm{~s} /$ frame; pixels, $256 \times 256$ ). (c) Enlarged AFM image of the square-shaped grating sample obtained without compensation for cross-talk between $\mathrm{X}$ - and Y-scanners (imaging rate 7 s/frame, pixels, $256 \times 256$ ). (e) Enlarged AFM image of the square-shaped grating sample obtained with compensation for cross-talk between $\mathrm{X}$ - and Y-scanners (imaging rate, 7 s/frame; pixels, $256 \times 256$ ).

Fig. 9 Simple fluorescence microscopy system installed in the sample stage-scanning HS-AFM setup. (a) A schematic illustration of the system. An arc lamp for fluorescence excitation and a CCD camera to capture bright-field and fluorescence images are installed in the bottom of the HS-AFM system. (b) An overlaid image of cantilever and 
fluorescence images. The white dotted line displays the outline of the cantilever's shadow. The white square with the arrow indicates the area size that the wide-area scanner can cover $\left(\sim 40 \times 40 \mu \mathrm{m}^{2}\right)$.

Fig. 10 HS-AFM images of B. subtilis captured using the wide-area scanner. (a) Topographic image of $B$. subtilis in a culture medium solution before the addition of lysozyme (imaging rate, $15 \mathrm{~s} /$ frame, pixels, $200 \times 200$ ). (b) Successive HS-AFM images showing bacteriolysis process of $B$. subtilis subjected to lysozyme. Lysozyme was injected at $t=240 \mathrm{~s}$ (imaging rate, $20 \mathrm{~s} /$ frame; pixels, $256 \times 256$ ).

Fig. 11 HS-AFM images of live COS-7 cells in response to insulin application. (a) Fluorescence image of a COS-7 cell transfected with mEGFP. The white dotted line indicates the outline of the cantilever's shadow. The white square indicates the area for HS-AFM imaging. (b), (c) HS-AFM topographical images of the same COS-7 cell as shown in (a) (b) before and (c) after the addition of insulin with the final concentration of $20 \mu \mathrm{g} / \mathrm{ml}$ into the observation solution (imaging rate, $10 \mathrm{~s} /$ frame; pixels, $200 \times 200$ ). The cell was starved with serum-free medium for $\sim 1$ hour before the experiments. (d), (e) HS-AFM images taken at the indicated times (d) before and (e) after the insulin addition. These images are overlaid with the corresponding images at $0 \mathrm{~s}$. The areas shown in green and magenta indicate newly appeared and disappeared structures in the entire imaged region, respectively, while the filled and open white arrows indicate newly appeared and disappeared structures at the leading edge of the cell, respectively.

Fig. 12 HS-AFM images of live hippocampal neurons cultured for 9 days in vitro. (a) Fluorescence image of a hippocampal neuron transfected with mEGFP. The white dotted lines show the outline of the cantilever's shadow. The white square indicates the area for HS-AFM imaging. (b) A sequence of HS-AFM topographical images of a dendrite captured for the area indicated in (a) (imaging rate, 5 s/frame; pixels, $200 \times$ 200). The white arrows indicate the growth and retraction of filopodia. The size of the dendrite is $\sim 400 \mathrm{~nm}$ in width and $\sim 200 \mathrm{~nm}$ in height. 
(a)

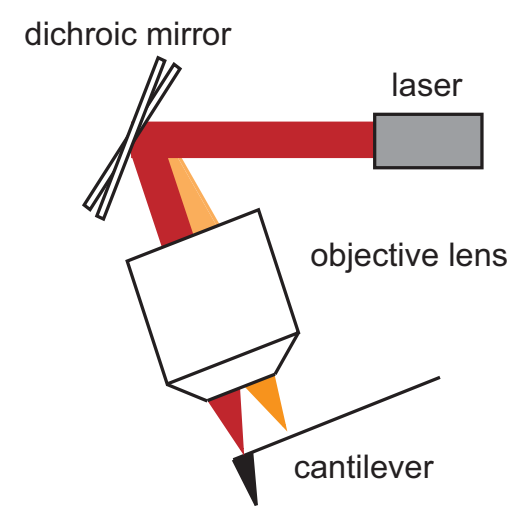

(d)

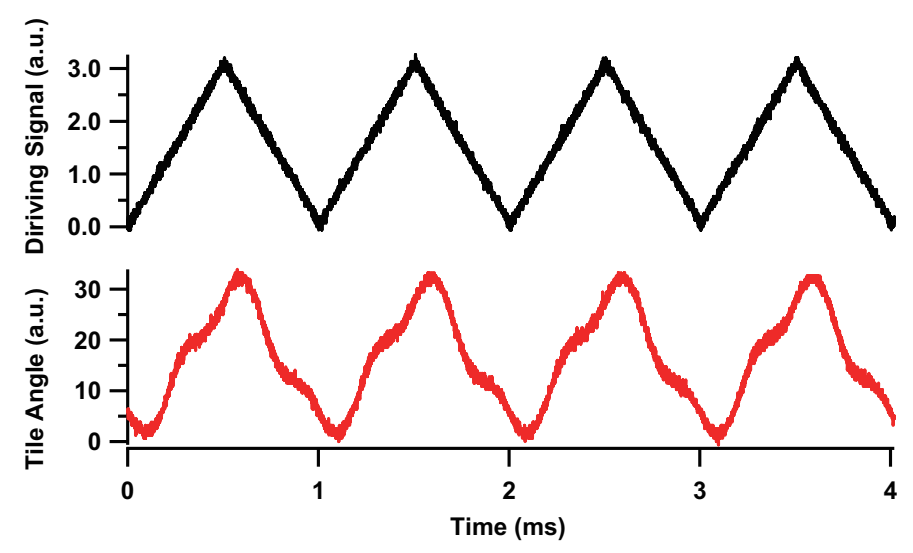

(b)

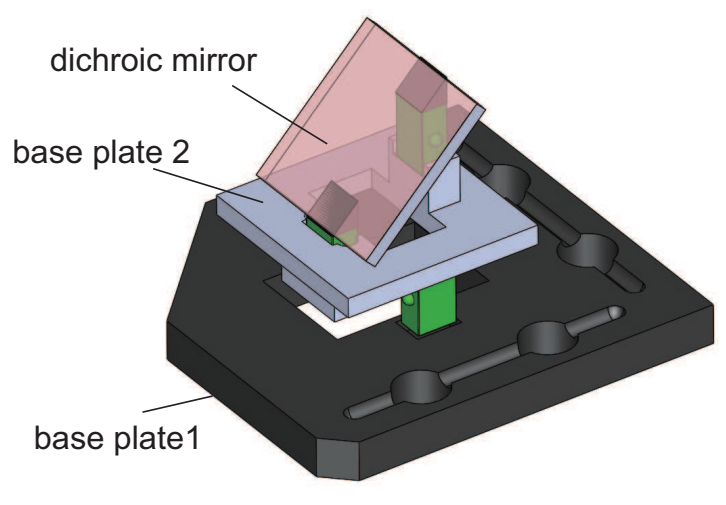

(c)

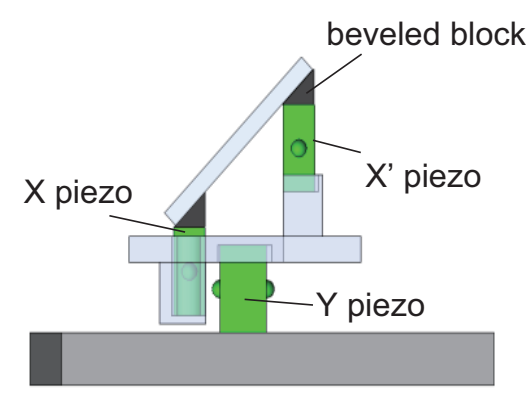

(e)
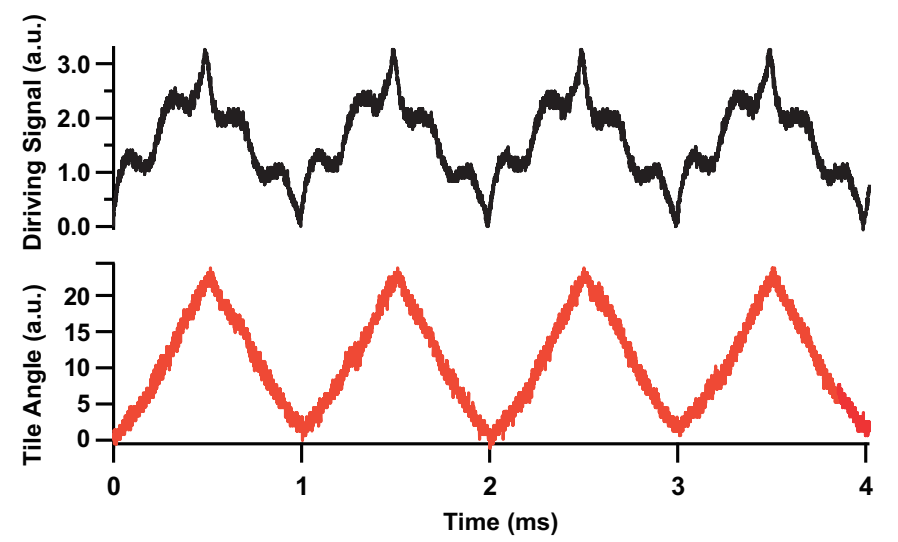

Fig. 1(2-column image) 
(a)

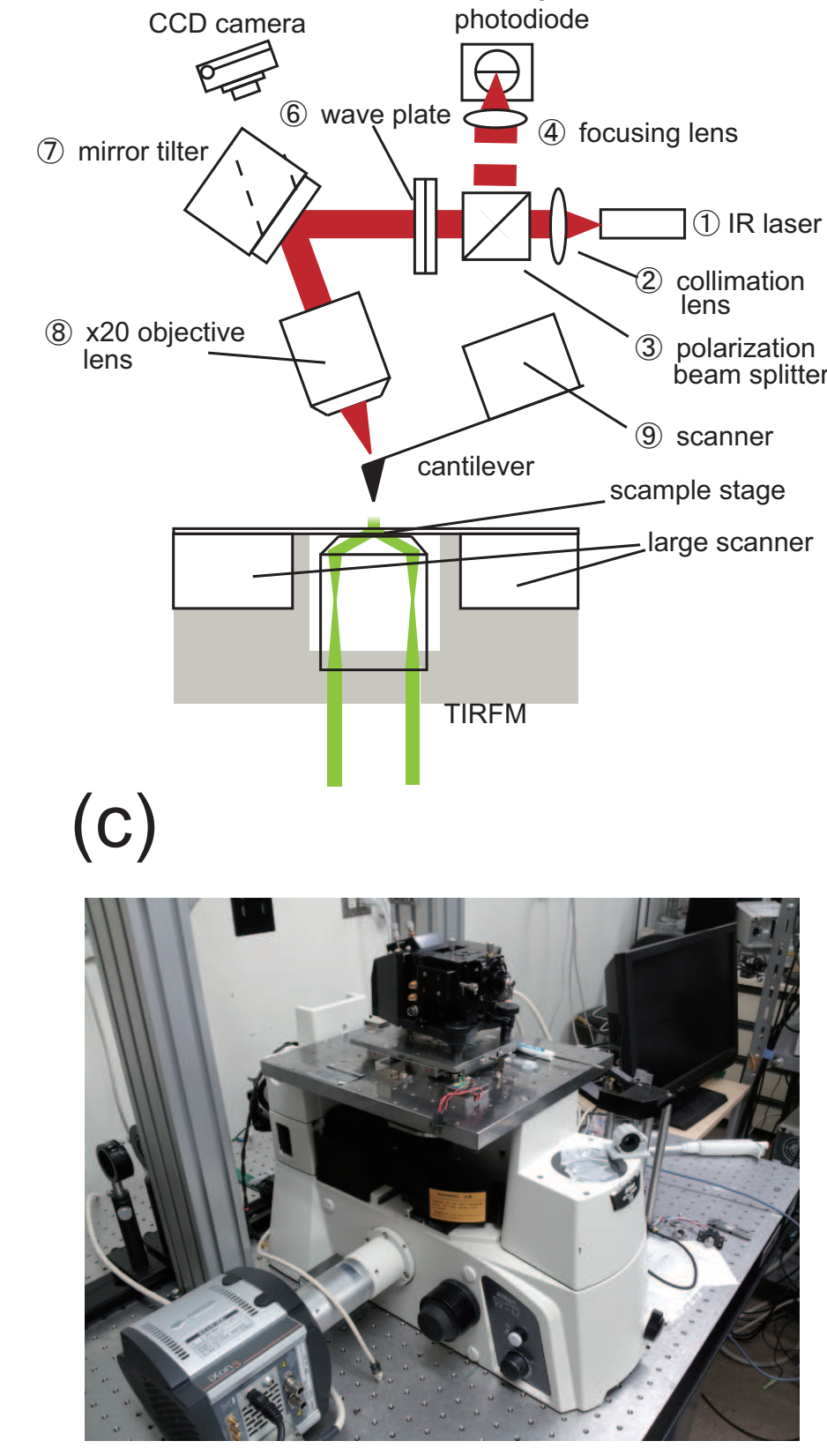

(b)
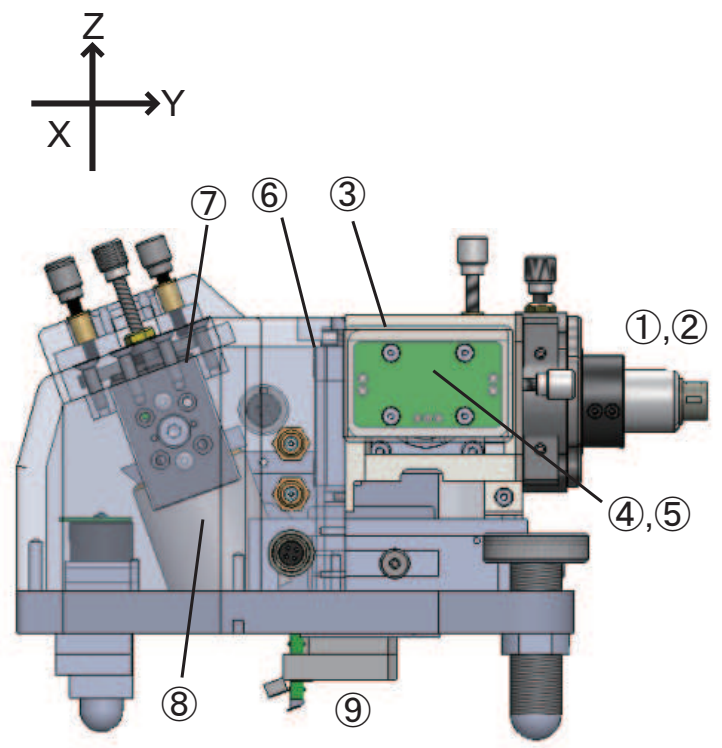

Fig. 3 (1.5-column image) 
(a)

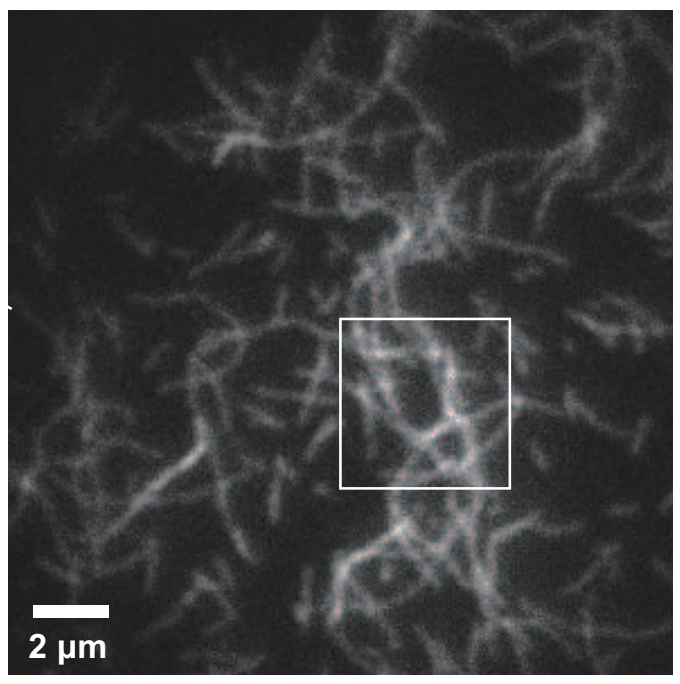

(c)

(d)
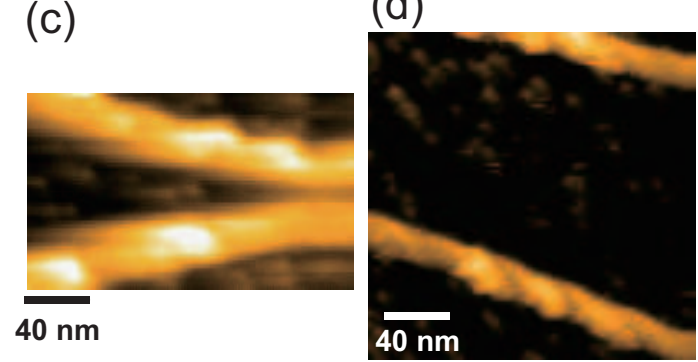

(b)

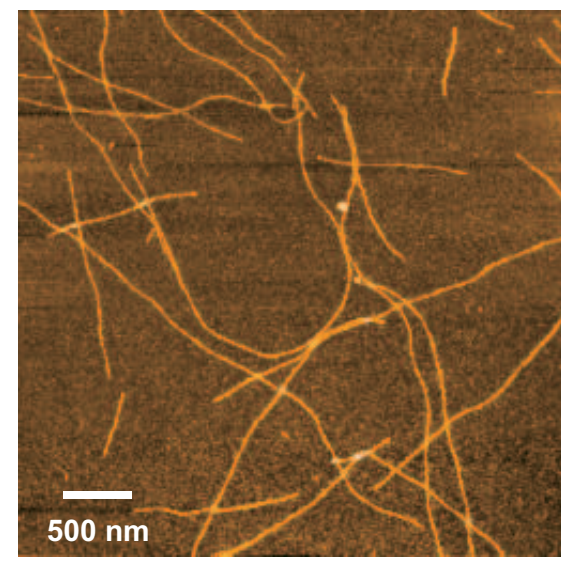

(e)

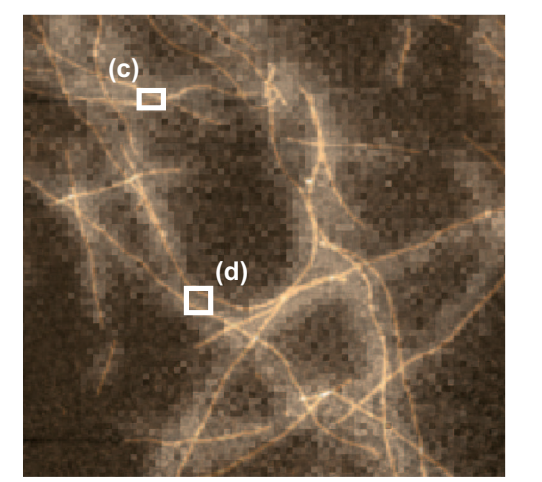

Fig. 4 (2-column image) 


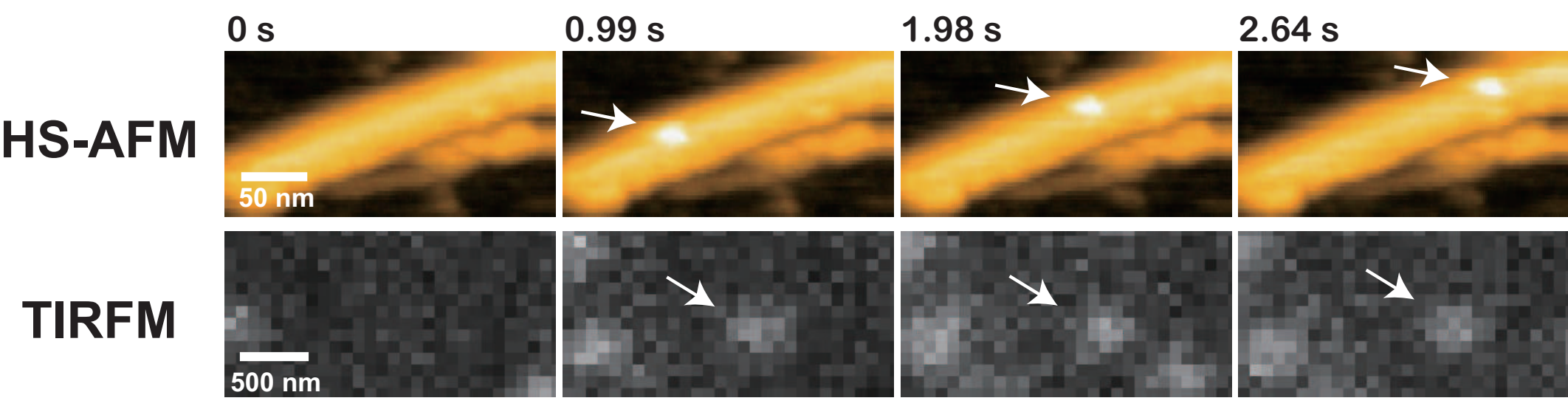

\section{Fig. 5 (2-column image)}

$500 \mathrm{~nm}$

$$
0.99 \mathrm{~s}
$$

$2.64 \mathrm{~s}$ 

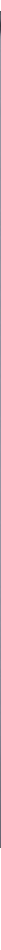

\section{Fig. 6 (1-column image)}


(a)

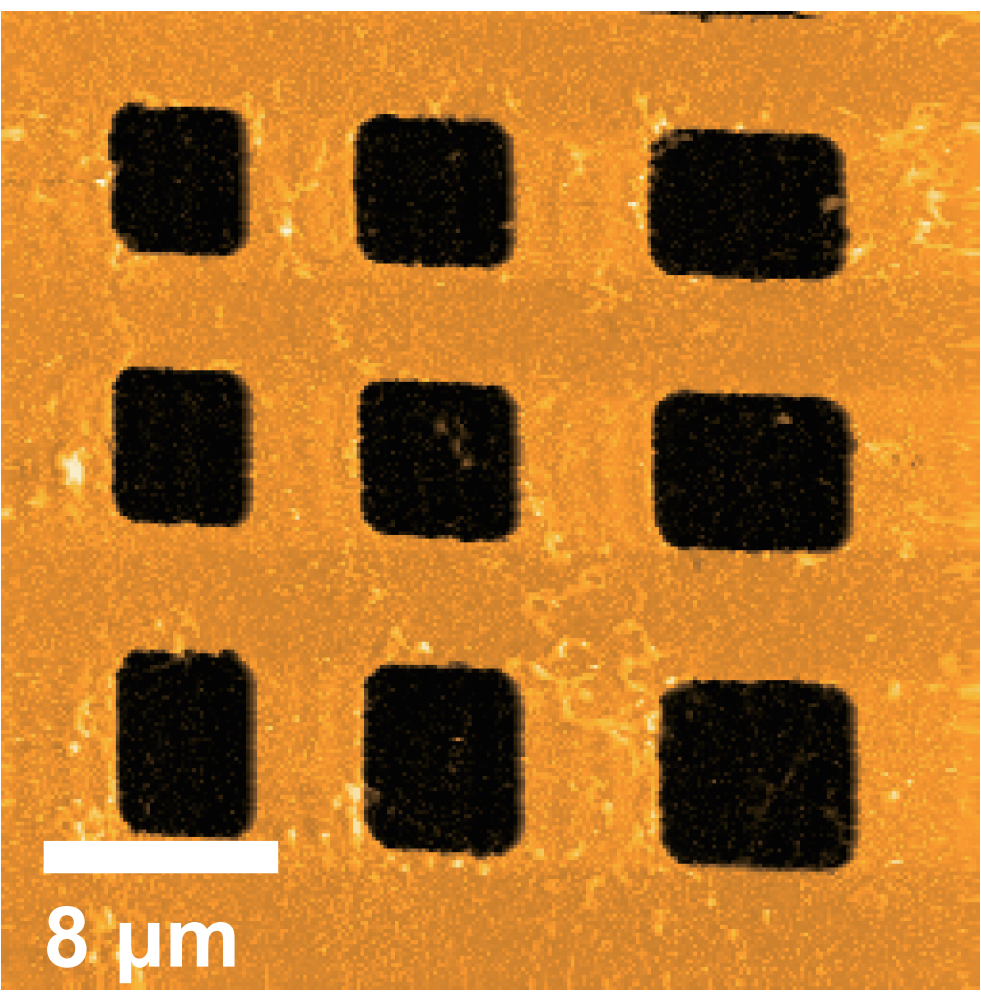

(c)

$3 \mathrm{\mu m}$ (b)

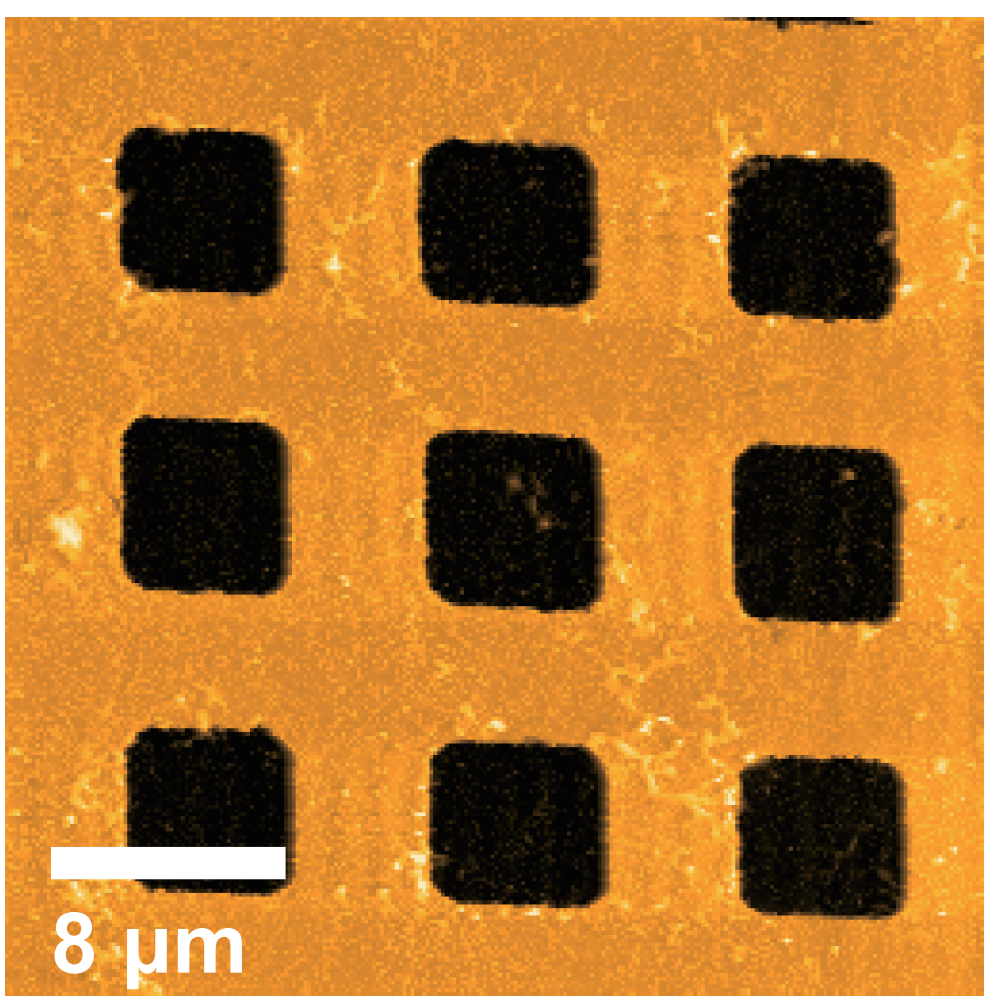

(d)

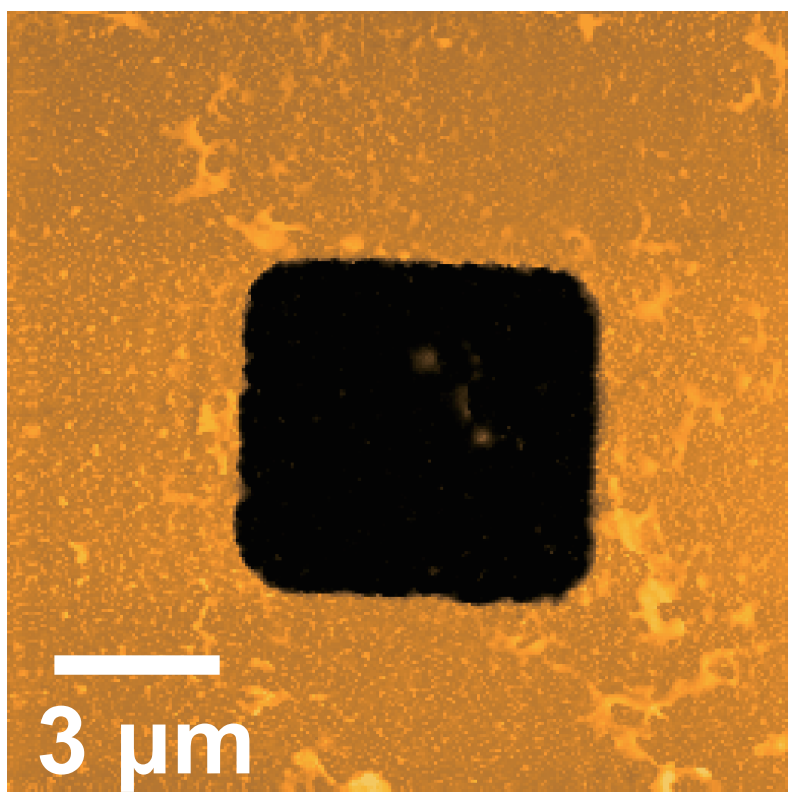

Fig. 8 (1-column image) 
(a)

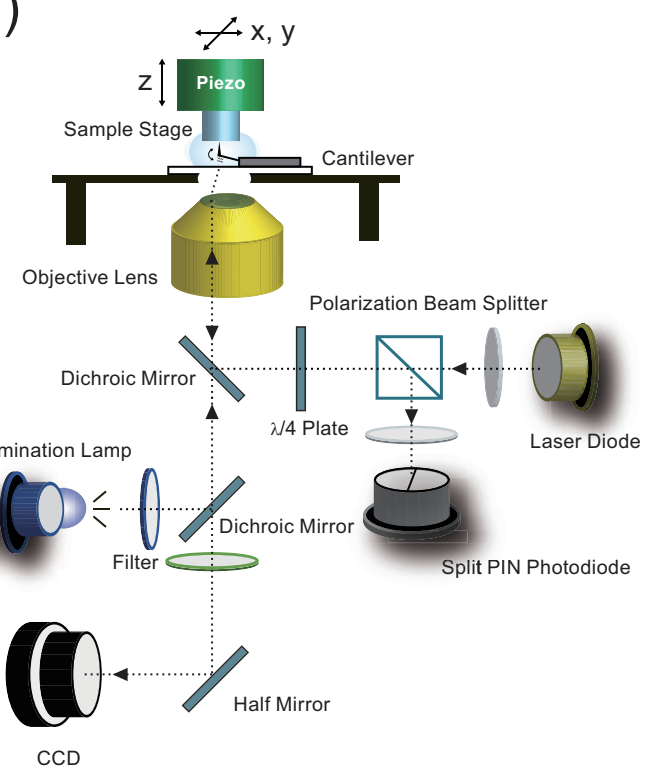

(b)

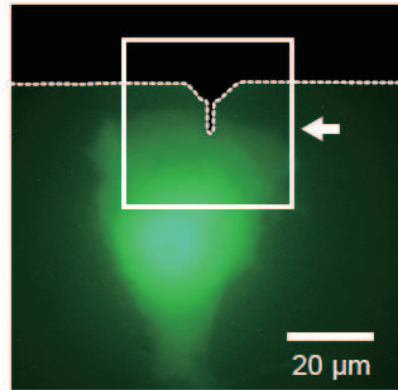

Fig. 9 (2-column image) 
(a)

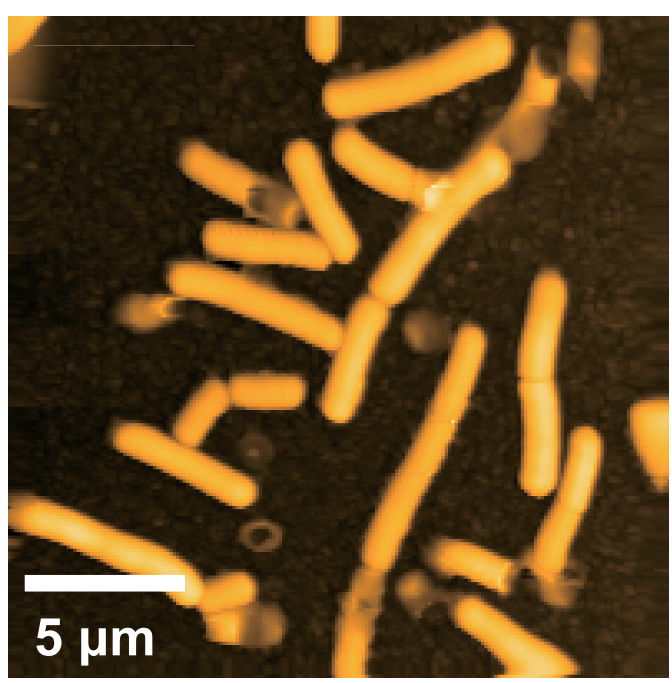

(b)

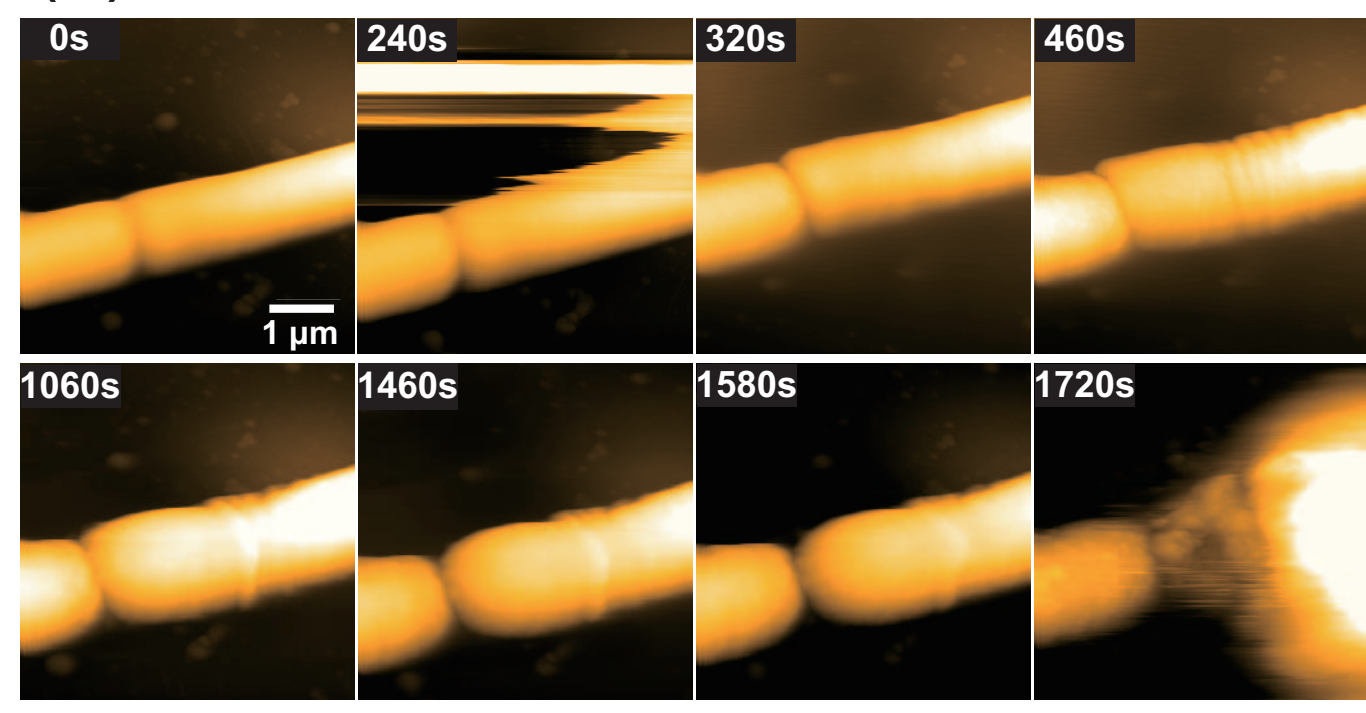

\section{Fig. 10 (2-column image)}


(a)

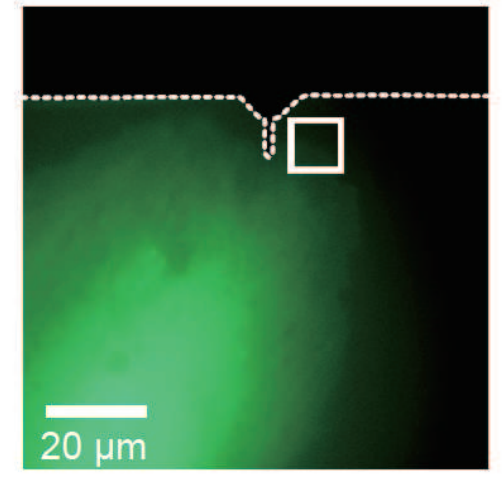

(b)

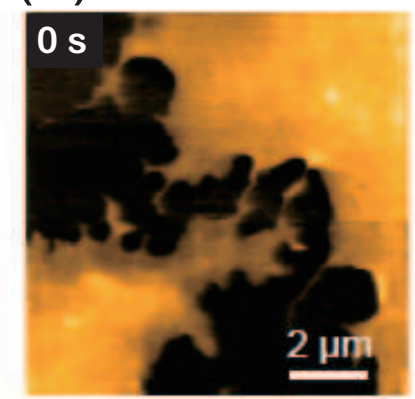

(c)

0

$\square$ (d)

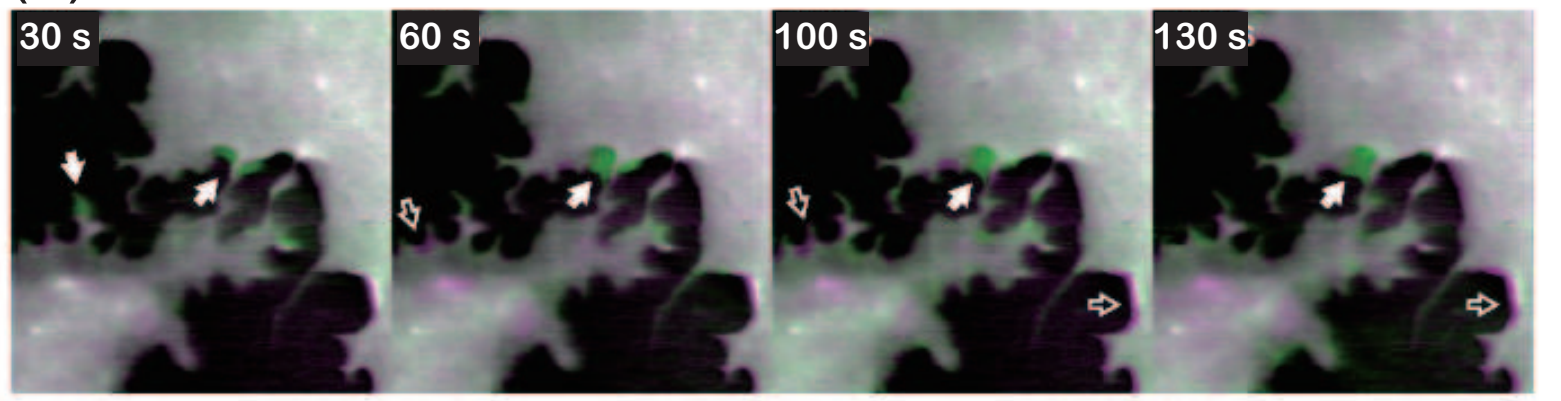

(e)

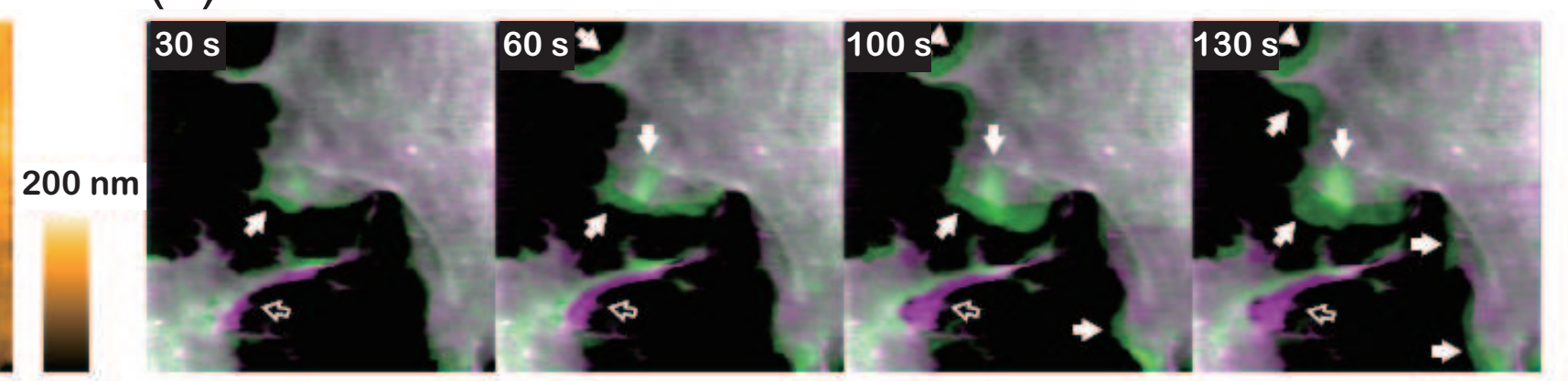

20

Fig. 11 (2-column image) 
